\title{
A High-Throughput Mechanical Activator for Cartilage Engineering Enables Rapid Screening of in vitro Response of Tissue Models to Physiological and Supra-Physiological Loads
}

\author{
Elisa Capuana ${ }^{a, b}$ Davide Marino ${ }^{a, b}$ Roberto Di Gesù b, c, d Vincenzo La Carrubba ${ }^{a}$ e \\ Valerio Brucato ${ }^{a}$ Rocky S. Tuan ${ }^{b, f}$ Riccardo Gottardi ${ }^{b, c}$, d

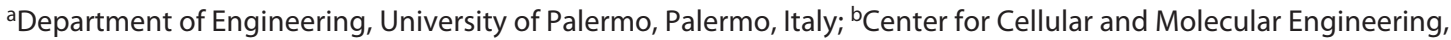 \\ Department of Orthopedic Surgery, University of Pittsburgh School of Medicine, Pittsburgh, PA, USA; 'Children's Hospital \\ of Philadelphia, and Department of Pediatrics, University of Pennsylvania School of Medicine, Philadelphia, PA, USA;

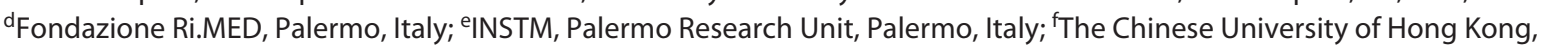 \\ Hong Kong SAR, China
}

\section{Keywords}

Cartilage $\cdot$ Mechanobiology $\cdot$ Mechanical activation .

Post-traumatic osteoarthritis $\cdot$ In vitro model

\begin{abstract}
Articular cartilage is crucially influenced by loading during development, health, and disease. However, our knowledge of the mechanical conditions that promote engineered cartilage maturation or tissue repair is still incomplete. Current in vitro models that allow precise control of the local mechanical environment have been dramatically limited by very low throughput, usually just a few specimens per experiment. To overcome this constraint, we have developed a new device for the high throughput compressive loading of tissue constructs: the High Throughput Mechanical Activator for Cartilage Engineering (HiT-MACE), which allows the mechanoactivation of 6 times more samples than current technologies. With HiT-MACE we were able to apply cyclic loads in the physiological (e.g., equivalent to walking and normal daily activity) and supra-physiological range (e.g., injurious impacts or extensive overloading) to up to 24 samples in one single run. In this report, we compared the early response of cartilage to physiological and supra-physiologi-
\end{abstract}

karger@karger.com www.karger.com/cto

Karger $\stackrel{\text { ' }}{5}$

GOPEN ACCESS
(C) 2021 The Author(s)

Published by S. Karger AG, Basel

This is an Open Access article licensed under the Creative Commons Attribution-NonCommercial-4.0 International License (CC BY-NC) (http://www.karger.com/Services/OpenAccessLicense), applicable to the online version of the article only. Usage and distribution for commercial purposes requires written permission. cal mechanical loading to the response to IL-1 $\beta$ exposure, a common but rudimentary in vitro model of cartilage osteoarthritis. Physiological loading rapidly upregulated gene expression of anabolic markers along the TGF- $\beta 1$ pathway. Notably, TGF- $\beta 1$ or serum was not included in the medium. Supra-physiological loading caused a mild catabolic response while IL-1 $\beta$ exposure drove a rapid anabolic shift. This aligns well with recent findings suggesting that overloading is a more realistic and biomimetic model of cartilage degeneration. Taken together, these findings showed that the application of HiT-MACE allowed the use of larger number of samples to generate higher volume of data to effectively explore cartilage mechanobiology, which will enable the design of more effective repair and rehabilitation strategies for degenerative cartilage pathologies.

(c) 2021 The Author(s)

Published by S. Karger AG, Basel

\section{Introduction}

Osteoarthritis (OA) is the most common degenerative joint disease [Thysen et al., 2015]. The development of $\mathrm{OA}$ is characterized by the structural degradation of articular cartilage accompanied by phenotypic changes in 
chondrocytes, pain, joint stiffness, and the progressive loss of mobility, that ultimately require joint replacement surgery for the restoration of function [Arokoski et al., 2000]. The etiology of OA is well defined only in approximately $12 \%$ of cases that are clearly post-traumatic [Carbone and Rodeo, 2017]. However, buildup of micro-traumas, obesity, and joint overuse are regarded as significant factors contributing to OA development and progression [Sauerland et al., 2003].

The physiological function of articular cartilage is load transduction and ensuring the smooth sliding of articular surfaces. Movement is also essential to tissue maintenance as physiological loading activates anabolic processes, resulting in increased extracellular matrix (ECM) synthesis and tissue remodeling, resulting in improved mechanical properties [Arokoski et al., 2000; Park et al., 2004]. However, overloading is deleterious and drives a catabolic response, which leads to tissue damage [Madej et al., 2016a]. The transition from pro-anabolic to procatabolic stress level is based on compression of the tissue, where physiologic strains are $\leq 20 \%$, while catabolic ECM degradation may start from $\geq 30 \%$ strains [Issa et al., 2018].

When undamaged, cartilage can slowly remodel in response to a wide range of biomechanical factors, mediated via a homeostatic balance between synthesis and degradation of its ECM: chondrocytes regulate this balance by sensing and responding to environmental factors, including mechanobiological stimuli, biochemical signals (e.g., cytokines and growth factors), and local matrix composition. In OA, however, the dynamic equilibrium between matrix degradation and repair shifts towards catabolism [Mueller and Tuan, 2008; Schätti et al., 2016].

Building on this evolving understanding of cartilage physiology, it is being more and more acknowledged that during in vivo joint loading, the mechanical environment comprises a complex combination of multiaxial compression, shear stress, and hydrostatic and osmotic pressure that all contribute to cellular response [Anderson and Johnstone, 2017]. New in vitro mechanical activation approaches have then focused on defining the role of shear and compressive loading on cartilage damage and repair [Frank et al., 2000; Schatti et al., 2011; Di Federico et al., 2014; Gottardi and Stoddart, 2018]. However, mechanistic in vitro research has mostly concentrated on static or dynamic mechanical compression as a starting point to model the metabolic response to loading in the regulation of the biosynthetic activity of articular cartilage [Mauck et al., 2007; Lujan et al., 2011; Luo et al., 2015; Anderson and Johnstone, 2017]. Irrespective of the load- ing approach, one of the major obstacles faced in these investigations has been the relatively low throughput of the in vitro mechanoactivation devices employed. In fact, the more controlled the load parameters were, the lower the number of samples that could be simultaneously activated. Scale up was either prohibitively time-consuming or required giving up control on the strain applied to individual cartilage samples [Mohanraj et al., 2014a, b].

To overcome some of these limitations, in this work we developed a High-Throughput Mechanical Activator for Cartilage Engineering (HiT-MACE) to apply individual dynamic compression to up to 24 cartilage samples in a single run. We first validated the accuracy of this new device by measuring the compressive and dynamic response of soft model materials and of cartilage tissue. Then, we used the HiT-MACE to deliver cyclic physiological and supra-physiological loading to cartilage explants and characterized the anabolic and catabolic mechanoresponsive activities of chondrocytes in their native matrix. Since supra-physiological loading could be an induction modality of post-traumatic OA, we further compared the induced catabolic pathways with that triggered by an acute inflammatory signal, i.e., interleukin (IL)-1 $\beta$. In this study, we specifically focused on the mechanoactivation of the transforming growth factor-beta (TGF- $\beta$ ) pathways, crucially involved in both cartilage development and OA [De Lise et al., 2000; van der Kraan et al., 2002; Santibañez et al., 2011; Tuan et al., 2013; Madej et al., 2014; van der Kraan, 2018]. Specifically, in the TGF- $\beta$ signaling pathway, activation of ALK $5 / \mathrm{Smad} 2 / 3$ triggers an anabolic, protective response, while ALK1/Smad 1/5/8 activation drives chondrocytes to terminal differentiation [Mariani et al., 2014]. Since the presence of active TGF- $\beta$ is known to modulate chondrocyte response to mechanical forces [Stevens et al., 2009; Madej et al., 2016b; Praxenthaler et al., 2018], in this work, TGF- $\beta$ was not added to the culture medium, to minimize perturbation of the specific pathway activation by physiological or supraphysiological loading.

\section{Materials and Methods}

HiT-MACE: Mechanical Components, Electronics, and Software

In the device, the base plate holding the multiwell plate can be actuated by two motors (MSX2, Velmex XSlide ${ }^{\mathrm{TM}}$ Assembly) along perpendicular directions ( $x$ and $y$ axis). Above this movable base plate, six compact linear actuators (L2018S0604-T3,5X1-25, NANOTEC) are positioned vertically and screwed on a platen to keep them in position during device operation (Fig. 1a). These linear actuators guarantee linear movements in the $z$ axis with a resolu- 


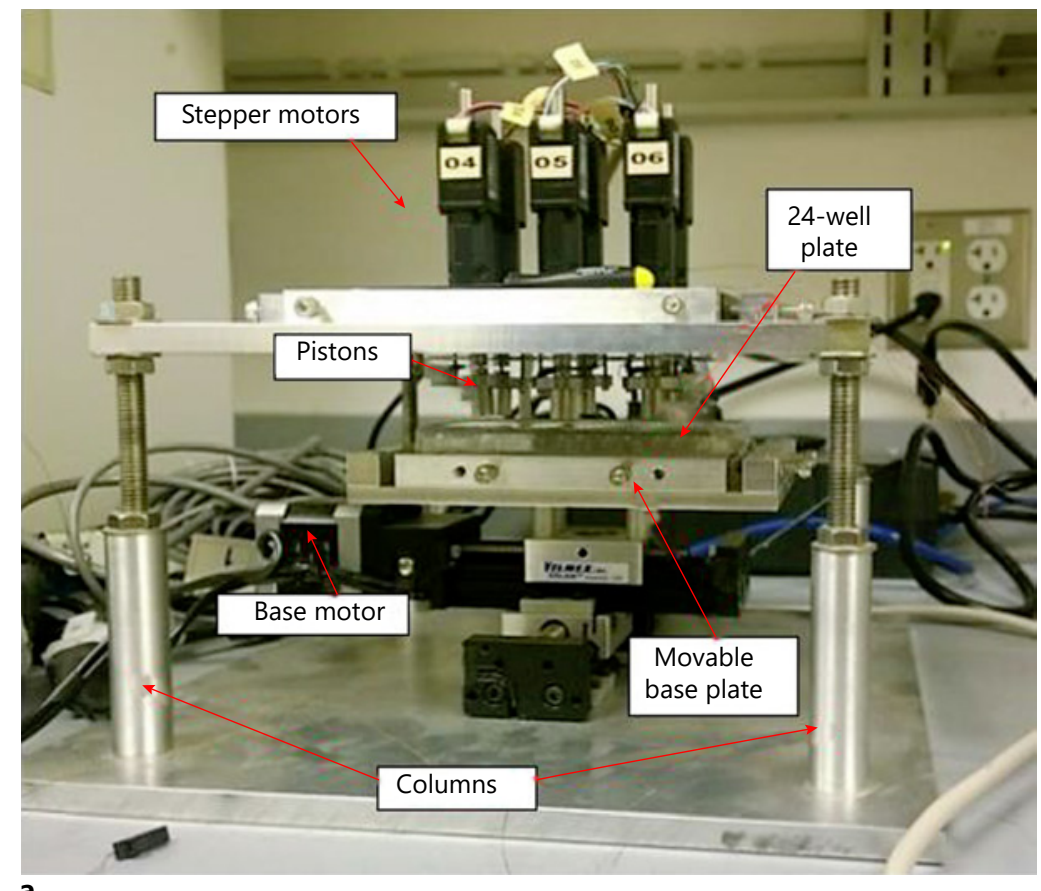

a

Fig. 1. a Final configuration of High Throughput Mechanical Activator for Cartilage Engineering (HiT-MACE). The base has the capability of moving in two orthogonal directions owing to two base motors and six compact linear actuators (resolution $5 \mu \mathrm{m}$ ) that are able to stimulate six wells at a time. Force is controlled and measured by the actuators and the voltage signal of six force sensors is amplified by six linear amplifiers. The complete system can fit inside a standard $\mathrm{CO}_{2}$ incubator and allows the stimulation of

tion of $5 \mu \mathrm{m}$ (step of each increment) and a peak thrust force of up to $40 \mathrm{~N}$, along with the capability of fully-automated format adjustment in the positioning system. Power and direction control of the six linear actuators are ensured by six Nanotech closed loop motor controllers connected to a data acquisition board (National Instruments, mod. 6210).

A force sensor (FlexiForce, mod. A301, Teskan Inc.) is attached at the end of each linear actuator to monitor applied loads in the $0-111 \mathrm{~N}(0-25 \mathrm{lb})$ force range. An inverting operational amplifier circuit acts as a current-to-voltage converter and provides the input to the data acquisition board contact feedback used to define the piston-specimen pre-load point-of-contact.

A software package created in LabView 11.0 (National Instruments) controls all motors and acquires both linear actuators and force-sensitive resistor (FSR) signals that are time-stamped, saved, and emailed to the user.

This instrumental setup operates in force feedback loop to identify piston-sample contact for each specimen, and in displacement control during mechanoactivation of the samples. The simple user's interface (Fig. 1b) allows one to define the threshold compressive force (voltage values) to define piston-specimen contact (zero reference position) for each motor. Prior to specimen activation loading, each piston moves until contact is reached, de-

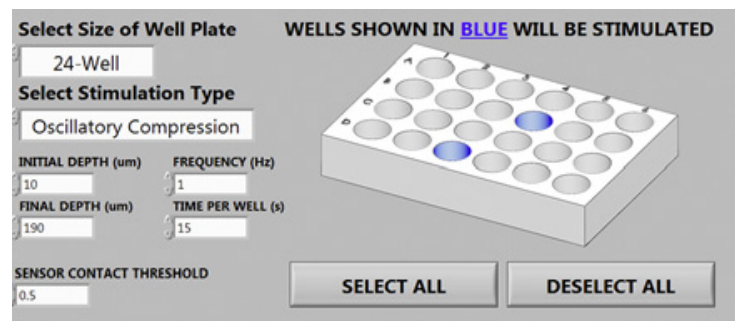

b

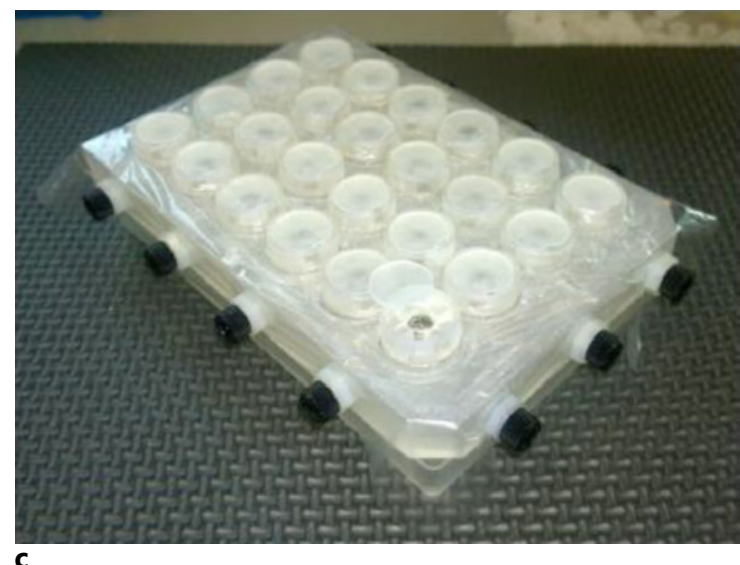

up to 24 native or engineered cartilage constructs through dynamic compression. $\mathbf{b}$ Graphic user interface for setting variables of the stimulation regimen (frequency and amplitude of oscillations, duration of loading, and threshold voltage ensuring piston-specimen contact). c Custom-designed 24-well plate lid: the force-sensitive resistors on the actuators push the flat buttons screwed to the internal pistons to induce semi-confined compression in an aseptic environment.

fining the individual $z$ axis zero position for individual samples, each having in principle a different height. Furthermore, the user can configure the maximum oscillation amplitude (distance covered by the pistons after contact) in relation to the maximum strain to be applied on the specimen and the cyclic loading frequency.

In summary, each piston identifies the point-of-contact (preload), then the compressive activation cycle starts from this reference position, performing loading over a six-samples group each cycle. Pistons are driven by triangular displacement waveform (displacement control), with user's defined frequency and amplitude (maximum strain). At the end of each cycle, the plate is repositioned over the next six-samples group by the MSX2 assembly to initiate the following loading run. The accuracy of this apparatus for modulus calculation was verified by comparing the equilibrium moduli of polydimethylsiloxane (PDMS) 20:1 and agarose $4 \%$ measured by HiT-MACE with that measured by an electromechanical tester ElectroForce ${ }^{\circledR} 3200$ Series III (TA Instrument, USA).

\section{Custom-Designed 24-Well Plate Lid for Aseptic Load}

Transduction

The mechanical stimulation of the cartilage specimens was performed using a custom-designed lid system (Fig. 1c) that allows load transduction from the piston to the specimens while, at the 


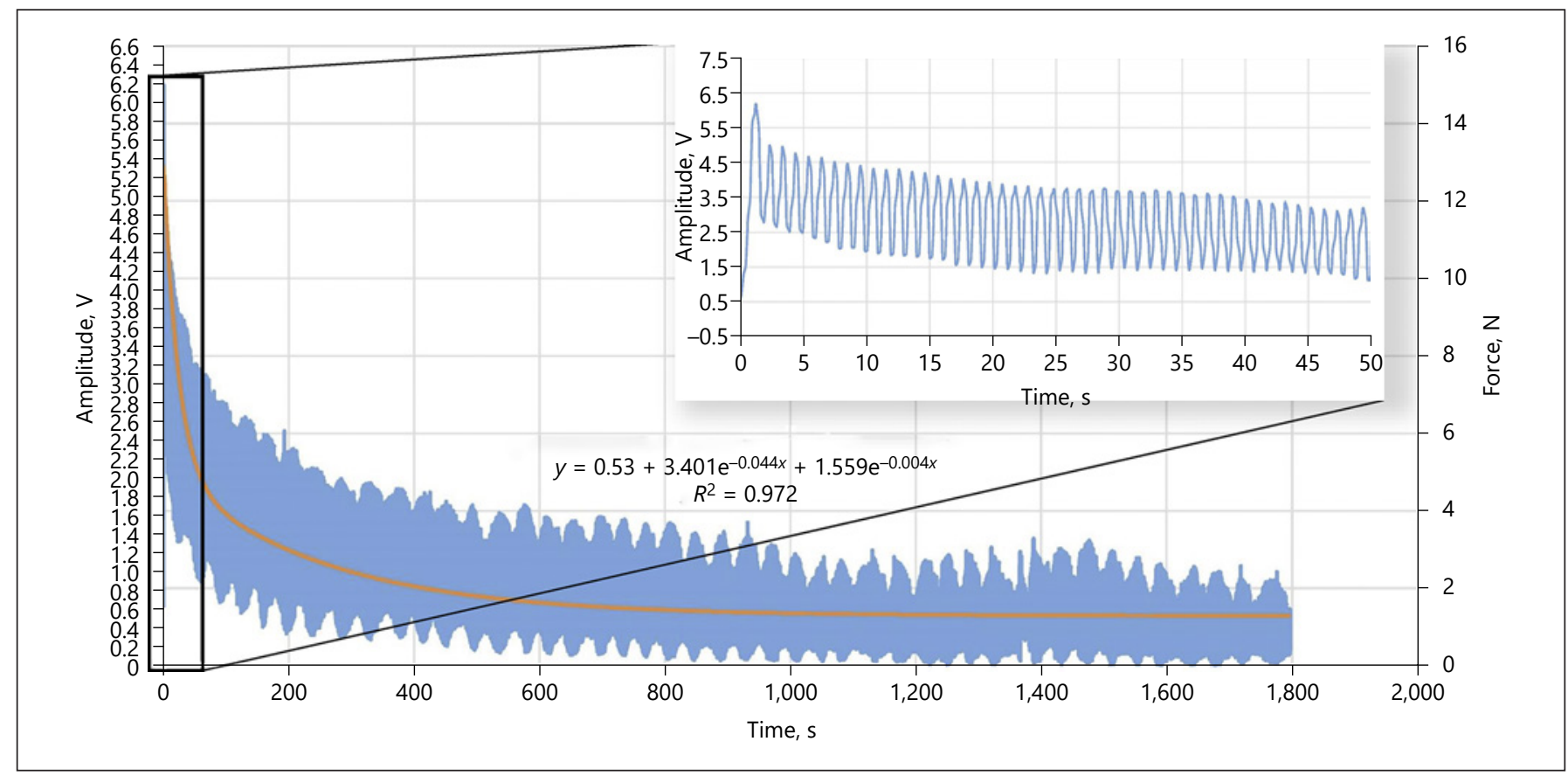

Fig. 2. A 1,800-s recording of force-sensitive resistor voltage measurements (left axis) and the corresponding applied force (right axis) fitted by a second-order exponential $\left(R^{2}=0.972\right)$ from dynamic compression $(1 \mathrm{~Hz}, 10 \%$ strain) of cartilage explants. The amplitude plateau $(0.53 \mathrm{~V})$, which is greater than $0.3 \mathrm{~V}$ (sensor

same time, maintaining sterility. First, holes were created in a 24well lid with the same footprint of each well. A Nylon membrane (McMaster-Carr, USA) with thickness of $0.05 \pm 0.01 \mathrm{~mm}$ was used to cover the holed lid. Through each hole, the membrane was screwed between a piston (inner side of the membrane towards the well) and a flat button (outer side of the membrane towards the linear actuators). To maintain the axial alignment of each piston, a system of guides was installed inside every well. Pistons, buttons, and the guides were modeled using Solidworks 2014 (Dassault, Systemes) and 3D printed via an EnvisionTEC Perfactory 4 printer by means of the WaterShed XC 11122 resin (DSM Somos, Heerlen, The Netherlands). Each part of the custom lid system can be sterilized in $70 \%$ isopropanol in water $(\mathrm{v} / \mathrm{v})$. To test the reliability of this apparatus for accuracy in mechanical evaluation, Young's moduli of PDMS 9:1 $(N=24)$ were calculated with and without this custom-made system and then compared.

\section{Force Sensor Calibration}

The FSRs were individually calibrated using an ElectroForce ${ }^{\circledR}$ 3200 Series III (TA Instrument, USA). Briefly, incremental loads were applied to each FSR recording the corresponding output voltage value. Data were processed by applying the following linear regression:

$$
F_{i}=C_{F S R} A+W_{i 0}
$$

where $A$ is the amplitude in volts, $W_{i 0}$ is the minimum detectable force in newtons, and $i$ is the FSR identifier.

Mechanical Activator for in vitro

Response of Cartilage to Loading contact threshold), ensures a preserved contact between the piston and the lid during the dynamic load. Inset Detailed display of the first 50-s recording illustrating the oscillation during dynamic loading, i.e., the pistons compress the samples to the set final depth and then oscillate around the setpoint.

\section{Preparation of PDMS Samples}

Cylindrical samples of PDMS were synthetized at the mass ratio of 20 or 9 parts Sylgard 184 (Dow Corning) silicone elastomer base and 1 part curing agent. After thorough mixing of the two compounds, the solution was degassed in a vacuum desiccator, then poured into the cylindrical molds $(9 \mathrm{~mm}$ diameter, $15 \mathrm{~mm}$ height) for the 20:1 ratio, or in a petri dish for the 9:1 ratio and cured overnight in a vacuum oven at $70^{\circ} \mathrm{C}$. Cylinders $(4 \mathrm{~mm}$ diameter, $3 \mathrm{~mm}$ height) were then punched out of the petri dish mold.

\section{Preparation of Agarose Samples}

Agarose hydrogels $(4 \%, \mathrm{w} / \mathrm{v})$ were prepared by mixing and heating $4 \mathrm{~g}$ of agarose powder (low melting point, Sigma-Aldrich) in $100 \mathrm{~mL}$ deionized water until complete dissolution. The solution was then poured into cylindrical molds ( $9 \mathrm{~mm}$ diameter, 15 $\mathrm{mm}$ height) and allowed to cool to room temperature. Finally, the gel was covered with a droplet of water to avoid dehydration.

\section{Real-Time Modulus Measurement with HiT-MACE}

Samples were placed in a 24 -well plate and compressed by applying displacement-controlled loading ( $10 \%$ strain) at a cycle frequency of $0.028 \mathrm{~Hz}$ for $30 \mathrm{~s}$. The contact threshold voltage of the FSR was set at $0.3 \mathrm{~V}$, greater than the maximum measured noise. Based on linear regression, the Young's modulus was calculated as the slope of the unloading portion (corresponding to the motors inversion to zero-position after axial compression) of the stress-strain curve. We employed only data from the unloading 


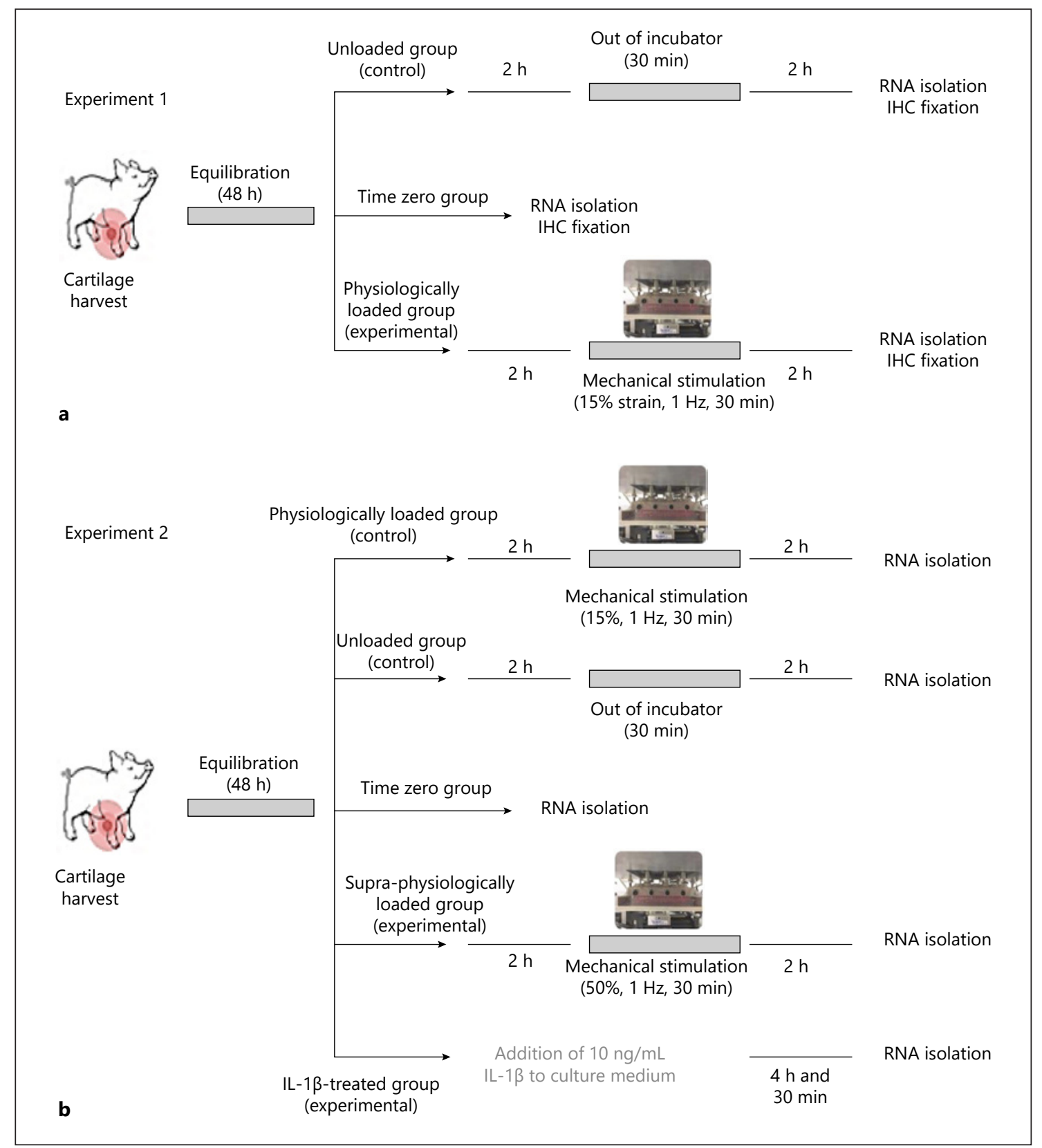

Fig. 3. Experimental design and timeline of physiological (a) and supra-physiological activation (b). a Loaded samples were mechanically activated with a $15 \%$ strain, $1-\mathrm{Hz}$ dynamic compression for $30 \mathrm{~min}$. b A $50 \%$ strain, $1-\mathrm{Hz}$ dynamic compression was applied to loaded samples for $30 \mathrm{~min}$. In both experiments, the me-

curves in order to exclude any possible contribution from plastic deformation [Stolz et al., 2004] and visco- or poroelasticity [Marklund et al., 2008; Taffetani et al., 2012] to the Young's modulus calculation. The stress $(\mathrm{MPa})$ was calculated from the voltage measurements converted to force $(\mathrm{N})$ (Equation 1) and from the sample contact surface area $\left(\mathrm{mm}^{2}\right)$, and the strain was obtained as the ratio between the linear displacement of each motor and the chanical stimulation was followed by $2 \mathrm{~h}$ of unloaded rest prior to sample processing. Additionally, in $\mathbf{b}$, the effect of IL- $1 \beta$ treatment ( $10 \mathrm{ng} / \mathrm{mL}$ of IL- $1 \beta$ added to culture media) on porcine chondrocytes gene expression was also examined.

initial height $(\mathrm{mm})$ of the specimen. As the system did not include a displacement encoder, a triangular wave matching the linear motor drive signal was used as the real-time motor position. The load (Equation 2) and the unload (Equation 3) real-time motor displacements are described by the following equations:

$$
\text { Displacement }{ }_{\text {load }}=\text { initial depth }+\frac{(\text { final depth }- \text { initial depth })}{\left(t_{\max }-t_{\text {in }}\right)} \times\left(t-t_{\text {in }}\right)
$$




$$
\text { Displacement }_{\text {unload }}=\text { final depth }-\frac{(\text { initial depth }- \text { final depth })}{\left(t_{\text {fin }}-t_{\text {max }}\right)} \times\left(t_{\text {fin }}-t\right), \text { (3) }
$$

where initial depth is the user's assigned initial position of the piston, final depth is the maximum amplitude of oscillation, $t_{\text {in }}$ is when the piston begins to interact with the sample, $t_{\text {fin }}$ is the final time of the mechanical loading, and $t_{\max }$ is the time corresponding to the force curve peak.

\section{Real-Time Modulus Measurement with Electromechanical} Tester

An ElectroForce ${ }^{\circledR} 3200$ Series III mechanical tester (TA instruments, formerly BOSE) with a $450-\mathrm{N}$ load cell was used for axial compression of each individual sample (10\% strain) at $0.1 \mathrm{~mm} / \mathrm{s}$ rate. Young's modulus was then calculated as described above using $10 \%$ for strain.

\section{Articular Cartilage Explants}

Full-thickness ( $1.4 \pm 0.23 \mathrm{~mm}$ height $)$ cylindrical cartilage plugs were harvested from the trochlea of the distal femur of porcine knee joints obtained from a local abattoir within 2 days of sacrifice and maintained on ice. Using a 4-mm biopsy punch, the explant discs were cored out perpendicular to the articular surface to obtain flat cylinders. After tissue isolation, all specimens were equilibrated at $37^{\circ} \mathrm{C}, 5 \% \mathrm{CO}_{2}$, and $95 \%$ humidity for $48 \mathrm{~h}$ with standard culture medium (Dulbecco's modified Eagle's media [DMEM] supplemented with $10 \%$ fetal bovine serum, and $1 \%$ penicillin/streptomycin/fungizone [PSF]) renewed daily before mechanical compression. For the mechanobiological characterization, the medium was switched to serum-free DMEM supplemented with $1 \%$ insulintransferrin-selenium (ITS+), $50 \mu \mathrm{g} / \mathrm{mL}$ ascorbic acid, and 2\% PSF. To simulate inflammatory culture condition, explants were incubated in the same medium supplemented with $10 \mathrm{ng} / \mathrm{mL} \mathrm{IL-1 \beta}$.

\section{Dynamic Relaxation Test}

Dynamic relaxation test was conducted using HiT-MACE under displacement control until reaching stress equilibrium. Readings to assess relaxation were acquired for $1,800 \mathrm{~s}$ with a $10 \%$ maximum strain amplitude and an oscillating frequency of $1 \mathrm{~Hz}$ to model gait. The relaxation curve (stress-time) was determined by fitting with a second-order exponential function (Fig. 2). Furthermore, the Young's modulus of the measured 9:1 PDMS and of the cartilage samples was measured right before and immediately after this long-term cyclic compression.

\section{Mechanobiological Experimental Design}

Following cartilage harvest and mechanical characterization, physiological and supra-physiological loading studies were carried out as outlined in Figure 3. In the first study (Fig. 3a), a total of 144 plugs from 8 different animals $(n=8)$ were randomly assigned as control and experimental groups, i.e., free-swelling/unloaded and loaded (15\% strain at $1 \mathrm{~Hz}$ ) conditions, respectively. In addition, a time zero ( $2 \mathrm{~h}$ before compression) group was included to normalize the RT-PCR data from treatment groups. Samples were harvested at $4 \mathrm{~h}$ and $30 \mathrm{~min}$, corresponding to a 2-hour interval after dynamic compression, and samples were analyzed by real time, RT-PCR and immunohistochemistry. Following this, supra-physiological loading and inflammatory investigations were completed simultaneously (Fig. 3b). The same time point and normalization group were used. As control, unloaded plugs (no IL-1 $\beta$ ) and samples physiologically compressed in the previous experiment ( $15 \%$ strain at $1 \mathrm{~Hz}$, no IL- $1 \beta)$ were identified. IL- $1 \beta$ treated (unloaded, $10 \mathrm{ng} / \mathrm{mL}$ of IL- $1 \beta$ ) and supra-physiologically loaded $(50 \%$ strain at $1 \mathrm{~Hz}$, no IL-1 $\beta$ ) plugs were used as experimental groups. For both test and control groups, the samples ( $N=80$, from $n=4$ donors $)$ were analyzed for gene expression by real-time RT-PCR.

\section{Dynamic Compressive Stimulation}

For both mechanical experiments, samples were placed into a 24-well plate, submerged in culture media and compressed for 30 min in groups of six specimens at a time. A 1-Hz cyclic frequency was used to simulate compression under a slow walking condition. The displacement-controlled and unconfined stimulation was performed by HiT-MACE in sterile conditions by means of the custom-designed lid apparatus.

\section{Immunohistochemical Analysis}

After 48 h of standard culture, 8 samples (2 per 4 donors) per condition were fixed overnight with $4 \%$ paraformaldehyde and stored at $4{ }^{\circ} \mathrm{C}$. Following dehydration in an ascending ethanol series $(25 \%, 50 \%, 70 \%, 95 \%$, and $100 \%)$, the samples were paraffinembedded and then sectioned at $5 \mu \mathrm{m}$ thickness. For immunohistochemical staining, the sections were de-paraffinized with HistoClear (National Diagnostics) and rehydrated in a descending ethanol series ( $100 \%$ to $50 \%$ ), and then gently washed under running cold tap water. Heat-mediated antigen retrieval was performed with sodium citrate buffer at $\mathrm{pH} 6.0$, for $20 \mathrm{~min}$ at $95^{\circ} \mathrm{C}$. Endogenous peroxidase was blocked at room temperature with $3 \%$ $\mathrm{H}_{2} \mathrm{O}_{2}$ (Sigma-Aldrich) in methanol for $10 \mathrm{~min}$, followed by suppression of nonspecific binding with $1 \%$ horse serum (Vector Labs) in PBS for $45 \mathrm{~min}$. The sections were then incubated with primary antibodies against SMAD-3 (Ab28379, Abcam) using a $1 / 100$ dilution overnight at $4^{\circ} \mathrm{C}$, washed three times with $\mathrm{PBS}$, and incubated with biotinylated secondary antibody following manufacturer directions (Vector Labs). Immunostaining was visualized by incubation with horseradish peroxidase streptavidin conjugate and a treatment with the Vectors NovaRED ${ }^{\mathrm{TM}}$ peroxidase substrate with hematoxylin QS (Vector Labs) counterstaining. As positive control for immunostaining, Michigan Cancer Foundation-7 (MCF7) breast cancer cells were used. The MCF-7 cells were seeded within a 6-well plate over a sterile cover glass at a density of $1.0 \times 10^{5}$ cells/ $\mathrm{mL}$, then incubated at $37^{\circ} \mathrm{C}$ and $5 \% \mathrm{CO}_{2}$ overnight. The adherent cells on coverslips were removed from the 6-well plate, fixed with $4 \%$ paraformaldehyde (Sigma-Aldrich) for $5 \mathrm{~min}$ at room temperature, incubated with primary antibodies against Smad-3 (Ab28379, Abcam), and treated as described for histological sections. Finally, samples were dehydrated, mounted with limonene mounting medium, and coverslipped. Images were acquired by a Nikon Eclipse E800 microscope outfitted with an Olympus DP74 camera.

\section{Analysis of Gene Expression}

Harvested cartilage explants, which were stored frozen in liquid nitrogen, were pulverized using mortar and pestle, and suspended and homogenized in TRIzol agent. Total RNA was extracted using RNeasy Mini kit (Qiagen) before suspension in RNase free water and quantified spectrophotometrically $\left(\mathrm{A}_{260}\right)$ using a NanoDrop ND-1000 spectrophotometer. mRNA from each sample was reverse transcribed into cDNA using SuperScript IV Reverse Transcriptase (Qiagen) according to the user manual. SYBR Green re- 
Table 1. PCR primers for porcine genes

\begin{tabular}{|c|c|c|c|}
\hline Genes & $\begin{array}{l}\text { Primer sequence } \\
\left(5^{\prime}-3^{\prime}\right)\end{array}$ & & $\begin{array}{l}\text { Amplicon length, } \\
\text { bp }\end{array}$ \\
\hline \multirow[t]{2}{*}{$A C T B$} & Forward & CACGCCATCCTGCGTCTGGA & 100 \\
\hline & Reverse & AGCACCGTGTTGGCGTAGAG & \\
\hline \multirow[t]{2}{*}{$A C A N$} & Forward & TTCCCTGAGGCCGAGAAC & 194 \\
\hline & Reverse & GGGCGGTAATGGAACACAAC & \\
\hline \multirow[t]{2}{*}{$A L K 5$} & Forward & ATGGGCTCTGCTTTGTCTCC & 206 \\
\hline & Reverse & TCCTGGAAAAGGACCAACAGT & \\
\hline \multirow[t]{2}{*}{$B M P 2$} & Forward & TGGAGGCTCTTTCAATGGAC & 204 \\
\hline & Reverse & CGAGGGCTGGGAAGAGGAGA & \\
\hline \multirow[t]{2}{*}{ COL2A1 } & Forward & GGATGGCTGCACGAAACACACCGG & 157 \\
\hline & Reverse & CAAGAAGCAGACAGGCCCTATG & \\
\hline \multirow[t]{2}{*}{ COL10A1 } & Forward & GCCCTTTTGCTGCTGCTATTGTC & 101 \\
\hline & Reverse & GTGTTGGATGGTGGGCCTTTTATG & \\
\hline \multirow[t]{2}{*}{ ID1 } & Forward & AGCTGAACTCGGAATCCCAA & 147 \\
\hline & Reverse & TTCAGCGACACAAGATGCGAT & \\
\hline \multirow[t]{2}{*}{$M M P 13$} & Forward & TGTTGGCTCATGCTTTTCCTC & 273 \\
\hline & Reverse & GGTTGGGGTCTTCATCTCCTG & \\
\hline \multirow[t]{2}{*}{ PAI1 } & Forward & СССТСТACTTCAACGGCCAG & 180 \\
\hline & Reverse & AGGGCAATTCCAGGATGTCG & \\
\hline \multirow[t]{2}{*}{ RLP4 } & Forward & AGGAGGCTGTTCTGCTTCTG & 185 \\
\hline & Reverse & TCCAGGGATGTTTCTGAAGG & \\
\hline \multirow[t]{2}{*}{ SMAD3 } & Forward & TGGTGCCACGCCACACAGAG & 213 \\
\hline & Reverse & TCGGGGAGAGGTTTGGAGAA & \\
\hline \multirow[t]{2}{*}{ TGFB1 } & Forward & GCACGTGGAGCTATACCAGAA & 101 \\
\hline & Reverse & CATCAAAGGACAGCCACTCC & \\
\hline
\end{tabular}

agent (Master Mix) was employed for RT-PCR using primer pairs designed for the target transcripts. Briefly, the reverse transcribed samples were subjected to 40 cycles of PCR using the StepOnePlus (Applied Bioscience) thermocycler with continuous fluorescence monitoring from each well of a Fast-Optical 96-well reaction plate. The results were quantified based on the cycle threshold $\left(\Delta \Delta \mathrm{C}_{\mathrm{T}}\right)$ method. For each gene, the final expression level was normalized to $\beta$-actin $(A C T B)$ as the housekeeping gene, which was found to be less affected by treatments of load or IL-1 $\beta$, compared to ribosomal protein L4 (RPL4). The results from each test group was then normalized to that from the corresponding control (timezero or no mechanical stimulation). We examined gene expression of aggrecan $(A C A N)$, transforming growth factor beta receptor 1 (ALK5), bone morphogenetic protein 2 (BMP2), collagen type II (COL2A1), plasminogen activator inhibitor 1 (PAI1), transforming growth factor 1 (TGFB1), SMAD3, collagen type X (COL10A1), inhibitor of DNA binding (ID1), and matrix metalloproteinase 13 (MMP13). The primer sequences for the target genes are shown in Table 1.

\section{Statistical Analysis}

All experiments were evaluated in the Graphpad software. The dataset normal distribution was evaluated by first running a Shapiro-Wilk test, the distribution was considered normal for $p \geq 0.05$. The data comparison was performed running a paired $t$ test or a one-way ANOVA followed by a post hoc Tukey test. Differences were considered significant if they reached a level of $p=0.05$.

\section{Results}

\section{Sensitivity and Linearity of Force Sensors}

To achieve real-time measurements of mechanical properties during the applied dynamic loading, force sensors were calibrated. The output voltage for each of the six FSR plotted against the measured applied load $(\mathrm{N})$ could be fitted by linear regression (online suppl. Fig. S1; see www. karger.com/doi/10.1159/000514985 for all online suppl. material). The slope of each calibration curve provided a calibration coefficient $\left(\mathrm{C}_{\mathrm{FSR}}\right)$ for each FSR and the y-intercept corresponded to the minimum detectable force. The latter had an average value of $170 \mathrm{mN}$, corresponding to a $0.15 \%$ strain for $4 \%$ agarose (Young's modulus $=1.77 \mathrm{MPa}$ ) or a $0.3 \%$ compression for a 20:1 PDMS (Young's modulus $=0.9 \mathrm{MPa}$ ), highlighting the high sensitivity of the FSRs for reliable mechanical measurements.

\section{Accuracy of Modulus Measurement without the Custom-Made Lid}

We determined and compared the Young's modulus values for different concentrations of agarose $(2 \%, 4 \%$, and $6 \%$ ) and PDMS (5:1, 10:1, and 20:1) using the HiT-MACE 
Table 2. Modulus values of biomaterials (PDMS 5:1, 10:1, and 20:1; 2,4 , and $6 \%$ agarose) measured by mechanical stimulators (HiTMACE and ElectroForce ${ }^{\circledR}$ tester)

\begin{tabular}{llll}
\hline Material & $\begin{array}{l}\text { HiT-MACE } \\
\text { measured } \\
\text { modulus, } \mathrm{MPa}\end{array}$ & $\begin{array}{l}\text { ElectroForce } \\
\text { tester measured } \\
\text { modulus, } \mathrm{MPa}\end{array}$ & $p$ value \\
\hline PDMS 5:1 & $1.45 \pm 0.13$ & $1.46 \pm 0.07$ & 0.742 \\
PDMS 10:1 & $2.45 \pm 0.37$ & $2.54 \pm 0.24$ & 0.329 \\
PDMS 20:1 & $0.90 \pm 0.17$ & $0.89 \pm 0.13$ & 0.82 \\
2\% agarose & $0.57 \pm 0.18$ & $0.58 \pm 0.04$ & 0.793 \\
4\% agarose & $1.60 \pm 0.38$ & $1.77 \pm 0.08$ & 0.042 \\
6\% agarose & $2.43 \pm 0.43$ & $2.89 \pm 0.12$ & 0.0001 \\
\hline
\end{tabular}

and a commercial ElectroForce mechanical tester. To maximize the accuracy in the Young's modulus measurement, we did not couple the HiT-MACE with the custom-made lid at this stage. As reported in Table 2, no significant differences $(p>0.05)$ were observed between the measurements obtained with the two devices for PDMS samples and $2 \%$ agarose. However, for $4 \%$ and $6 \%$ agarose the modulus values were significantly different suggesting a lower accuracy in the HiT-MACE, probably caused by a small slip between sensors and samples. However, for these agarose compositions, Table 2 shows that the mean values of the moduli measured with each device are very close in magnitude although statistically different. Notably, the stressstrain loading-unloading curves (Fig. S2) for both devices showed no hysteresis for PDMS and significant hysteresis for agarose, as could be expected for a material with high water content and porosity with associated higher dissipation energy [Stolz et al., 2004]. Overall, these findings suggested an acceptable accuracy of the customized mechani$\mathrm{cal}$ activator to measure the elastic modulus of materials in the typical ranges of cartilage ( 1 MPa) [Li et al., 2003].

\section{Effect of Application of Custom-Made Lid on Modulus Measurement}

A custom-designed 24-well plate lid was fabricated to ensure sterility during mechanical activation of biological samples. We tested how much the presence of the lid apparatus affected the evaluation of Young's modulus by measuring PDMS 9:1 plugs $(N=24)$ with the HiT-MACE with and without the lid. Sample dimensions were chosen to closely mimic the cartilage explants we typically test: 3 $\mathrm{mm}$ thickness as that of articular cartilage and $4 \mathrm{~mm}$ diameter typical of our porcine explant. PDMS modulus has been reported to peak at a 9:1 mixing ratio [Johnston et al., 2014], which we used for this initial assessment.

Mechanical Activator for in vitro

Response of Cartilage to Loading
Stress-strain data showed a significant change $(p<$ $0.0001)$ in moduli (3.6 $\pm 0.5 \mathrm{MPa}$ without the lid, $10.47 \pm$ $4.39 \mathrm{MPa}$ with the lid) caused by the friction between the lid piston and its guide. Overall, each well of the lid-plate assembly displayed a typical dissipative force; hence, changes in modulus value between either different conditions or time points or biomaterials could in principle still be inferred. However, as the goal of this study was not to accurately determine modulus of biological constructs, the lid-plate assembly should be applicable for the delivery of dynamic compression and analysis of its effect on cartilage biology.

\section{Dynamic Relaxation}

During dynamic compression both cartilage and PDMS relaxed over time. We used a second-order exponential decay function to fit the dynamic relaxation data for PDMS 9:1 and cartilage plugs (Fig. 2 and online suppl. Fig. S2-3) with notable goodness of fit: $R^{2}=0.984$ and $R^{2}=0.972$, respectively.

For the dynamic parameters used in this test, cartilage reached a zero steady-state stress at around $800 \mathrm{~s}$, whereas PDMS exhibits a nonzero equilibrium even after 3,600 s. According to polymer dynamics theory, relaxation proceeds more slowly with decreased polymer mobility [June et al., 2009] while fluid flows out of the matrix. Under these slow-frequency loads, PDMS and cartilage exhibited different viscoelastic behaviors, with a viscous-like and elastic-like response, respectively, extensively described by the biphasic model of articular cartilage [Cohen et al., 1998; Anderson and Johnstone, 2017] and polymer dynamics [Cespi et al., 2007]. Comparing the Young's modulus values before and after relaxation showed no significant change in PDMS stiffness, consistent with a small change in stress from the peak to the equilibrium that induces small changes in modulus. The cartilage modulus instead increased (from 15.7 $\pm 3.4 \mathrm{MPa}$ to $18.2 \pm 5.3 \mathrm{MPa}$; $p<0.05$ ), consistent with the biphasic theory that predicts increased stiffness as a consequence of decreased tissue volume during relaxation and increased interstitial pressurization [Park et al., 2004; Anderson and Johnstone, 2017; Lawless et al., 2017]. However, it should be noted that the reported cartilage moduli were not "real" values as they were affected by the friction between piston and guides. Nevertheless, this frictional contribution was constant and the difference in modulus before and after dynamic compression could be considered realistic.

Importantly, this 1-hour-long dynamic compression scheme demonstrated that the apparatus could mechanically activate cartilage explants for an extended period of 


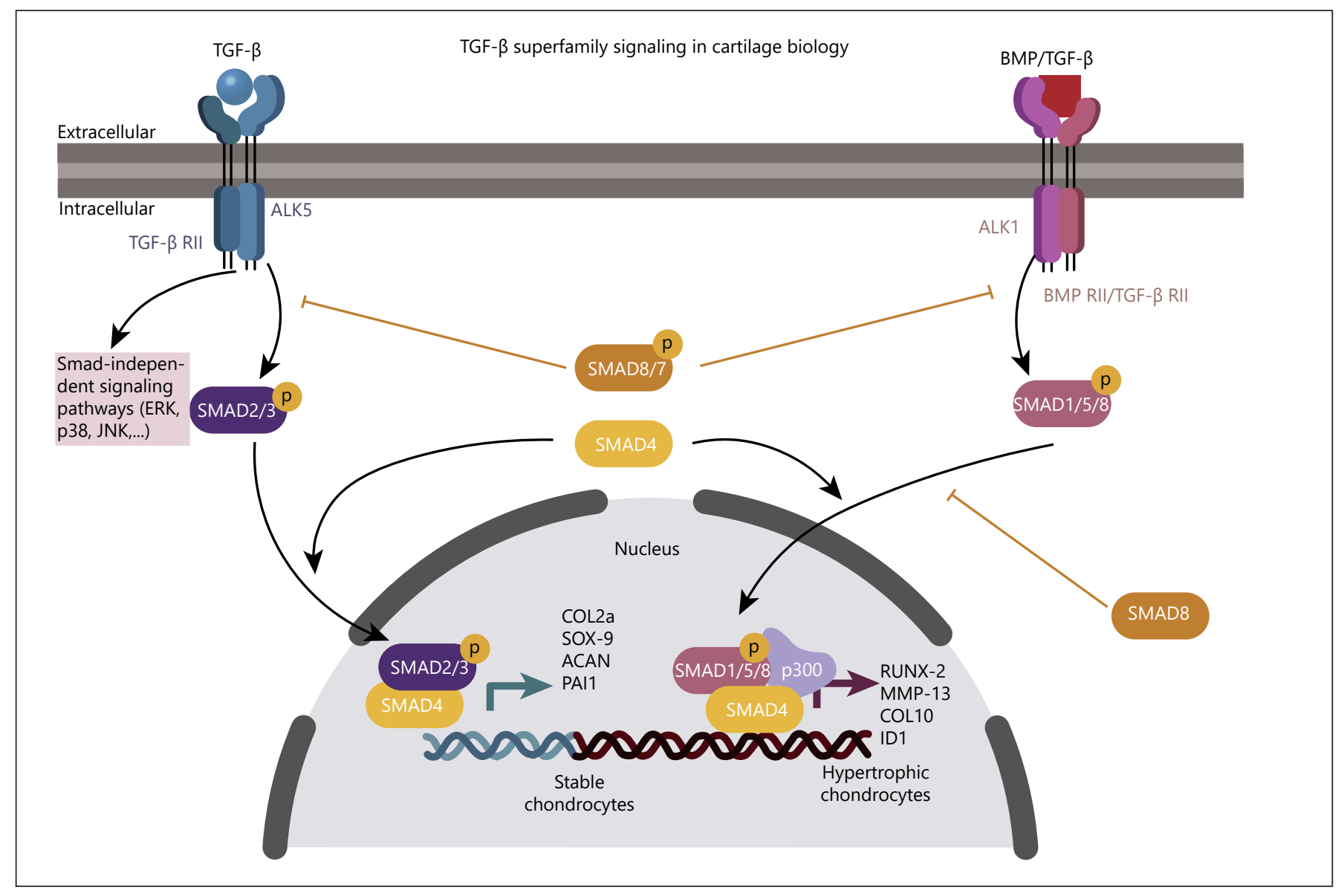

Fig. 4. Diagrammatic depiction of TGF- $\beta$ superfamily signaling pathway known to be linked to the growth and differentiation of chondrocytes, as well as to repair and inflammation processes after cartilage injury. The $\operatorname{Smad} 2 / 3$ signaling route, activated by ALK5 receptor I/TGF- $\beta$ binding, regulates articular cartilage repair and homeostasis. The ALK1 receptor I pathway stimulates SMAD1/5/8 route inducing terminal chondrocyte differentiation and $M M P 13$ expression.

time (twice that used in the tests described below) without sample breakage or sliding during the period of cyclic compression while maintaining contact between the FSRs-connected piston and the samples.

\section{Short-Time Dynamic Physiological Compression of}

Cartilage Activates Anabolic TGF- $\beta$ Signaling

Physiological strain is in the $\leq 20 \%$ range and is pro-anabolic while transition to a pro-catabolic response occurs for $\geq 30 \%$ supraphysiological strain [Issa et al., 2018]. To investigate the influence of physiological mechanical forces on cartilage explants, dynamic compression was applied for $30 \mathrm{~min}$ at $15 \%$ strain. After this activation, the expression levels of early signaling events downstream of the receptors and of mechanoresponsive target genes were analyzed to assess whether a direct crosstalk existed between mechano- transduction and TGF- $\beta$ signaling cascades (Fig. 4). Out of the 9 monitored genes, 7 showed statistically significant ( $p>0.05$ ) upregulation after loading with respect to the unloaded controls (Fig. 5). Gene expression levels were normalized to that of the housekeeping gene $A C T B$, previously shown to be least affected by mechanical activation of the tissue [Al-Sabah et al., 2016].

Consistent with previous findings, a 15\% dynamic compression, i.e., in the physiological loading range, upregulated both $A C A N$ [De Croos et al., 2006] and COL2A1 [De Croos et al., 2006] as well as SMAD3, BMP2, ALK5, and TGFB1 (up to 2 -fold) compared to $t=0$, suggesting that physiological loading activated the TGF- $\beta$ pathway. ID1, a gene downstream of Smad 1/5/7 signaling and one of the immediate targets of TGF- $\beta$ [Liang et al., 2009] and BMP [Wang et al., 2014], was also upregulated (Fig. 6). 


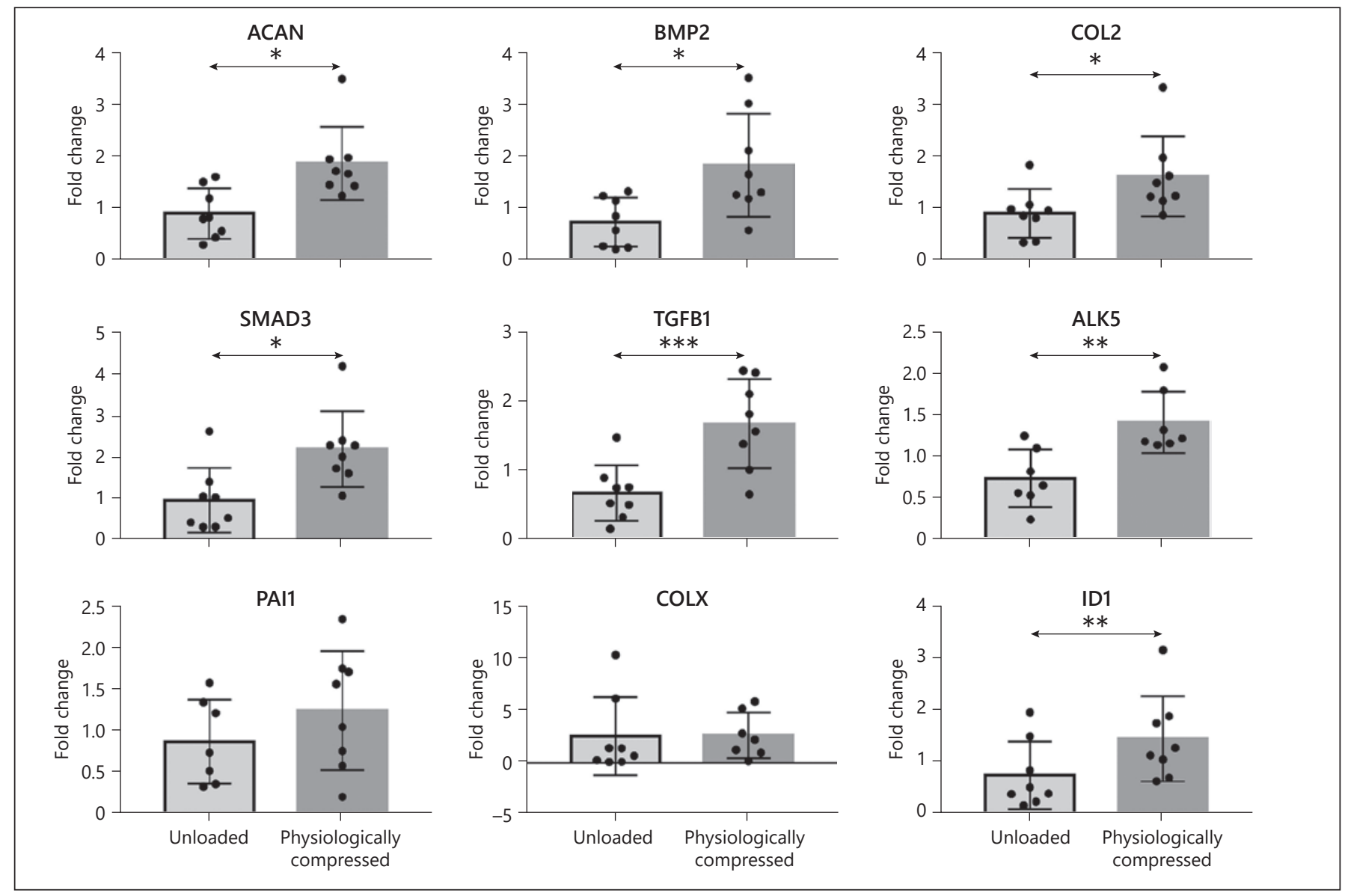

Fig. 5. Changes in gene expression profile in cartilage explants subjected to physiological loading samples at $2 \mathrm{~h}$ after $30 \mathrm{~min}$ of loading. All gene expression levels are expressed relative to that of controls before loading. Anabolic TGF- $\beta$ signaling was enhanced by physiological compressive loading, as suggested by the upregulation of $A L K 5$ and SMAD3, consistent with early prevention of chondrocyte hypertrophy. Data were analyzed using a paired $t$ test $\left(n=8\right.$ donors; statistical significance assigned at ${ }^{*} p<0.05,{ }^{* *} p<0.01$, and $\left.{ }^{* * *} p<0.001\right)$.

Furthermore, the non-Smad pathways involving COL$10 A 1$ was unaffected by mechanical activation, which was not surprising given the known late marker nature of collagen type X. Finally, the rapid induction of SMAD3 by physiological compression was confirmed by immunostaining (Fig. 7), showing no staining for $t=0$ and unloaded controls (Fig. 7a, b) but strong Smad 3 staining in compressed explants, especially in the cartilage upper layers (Fig. 7c) (MRF-7 cells that highly express Smad 3 were used as positive control for immunostaining, Fig. $7 d$ ).

\section{Short-Time Dynamic Supra-Physiological}

Compression Activates a Protective Response to

Inhibit Cartilage Damage

In vitro treatment with pro-inflammatory cytokines or exposure to supra-physiological mechanical loading or impact have been used as disease inducers in OA models [Fitzgerald et al., 2004; Torzilli et al., 2011; Alexander et al., 2013; Bonnevie et al., 2015; Occhetta et al., 2019]. To compare these two approaches, we repeated the same test described above by running the HiT-MACE twice, but this time applying first physiological loading (15\% strain) on one set of samples ( $n=4$ donors), and next supra-physiological loading (50\% strain) on a second set of samples ( $n=4$ donors). To determine how a short-time supraphysiological dynamic loading compared to a pro-inflammatory stimulation, we also included a test group of unloaded cartilage exposed to IL- $1 \beta$ for the same time as the loading, with analysis of gene expression profiles that included MMP13, known to be upregulated in OA (Fig. 6).

As expected, the physiologically activated and unloaded samples exhibited a gene profile similar to the observa- 
tions from the previous experiment (Fig. 5). In particular, the statistically significant increase of $A C A N, S M A D 3$, TGFB1, and ID1 was confirmed in physiologically compressed samples even with $n=4$, while the upregulation of ALK5, BMP2, and COL2 did not yet reach significance $(p=0.06)$, but presented a strong trend following the same pattern shown in Figure 5. Considering that there were 4 donors in this test while in the previous one there were 8 , this was not surprising and a slightly larger sample size would have likely achieved $p<0.05$.

Compared to the static condition, supra-physiological loading induced marked increases in ALK5, TGFB1, and $B M P 2$ levels, while SMAD3 ( $p=0.08), I D 1(p=0.08)$, and $A C A N(p=0.06)$ expression showed an increasing trend that did not yet reach significance, but still suggesting activation of the Smad3/TGF- $\beta$ pathway. Pro-catabolic genes MMP13 and COL1OA1 were not upregulated after 30 min of supraphysiological loading, thus not matching hypertrophic characteristics detected in advanced OA.

Notably, the gene expression levels for ACAN and $S M A D 3$ were 2 -fold higher for physiological loading compared to supra-physiological compression $(p<0.05)$, while TGFB1 $(p=0.09)$ and COL2A1 $(p=0.06)$ only trended to be more upregulated by $15 \%$ compressive strain, and ALK5 $(p=0.06)$ in the opposite direction by $50 \%$ strain, suggesting that the anabolic response onset was more pronounced by physiological load condition [Fitzgerald et al., 2004; Madej et al., 2014; Issa et al., 2018].

When IL- $1 \beta$ was added to the culture media under static conditions, the expression of ALK5 (2-fold) and BMP2 (23-fold) was significantly increased compared with the unloaded controls. Furthermore, expression of the pro-inflammatory gene $M M P 13$ also showed an increase in trend $(p=0.07)$ although the broad spread of data points prevented reaching significance. While $B M P 2$ and ALK5 expression were upregulated in both supraphysiological loading and pro-inflammatory stimulation, IL- $1 \beta$ induced a more marked response. Conversely, expression levels of ACAN, SMAD3, TGFB1, COL10A1, and ID1 were lower in IL-1 $\beta$ stimulation compared to physiologically compressed samples, whereas ALK5 and BMP2

Fig. 6. Gene expression profiles in cartilage explants subjected to physiologically loaded, supra-physiologically loaded, and IL-1 $\beta$ treated groups. All gene expression levels are expressed relative to that of controls before loading. The results suggest that, compared to treatment with IL-1 $\beta$, exposure to an excessive, supra-physiological load appears to initiate a self-protective response in tissue repair. Data were analyzed using a paired $t$ test $(n=4$ donors; statistical significance assigned at $* p<0.05$ and $\left.{ }^{* *} p<0.01\right)$.
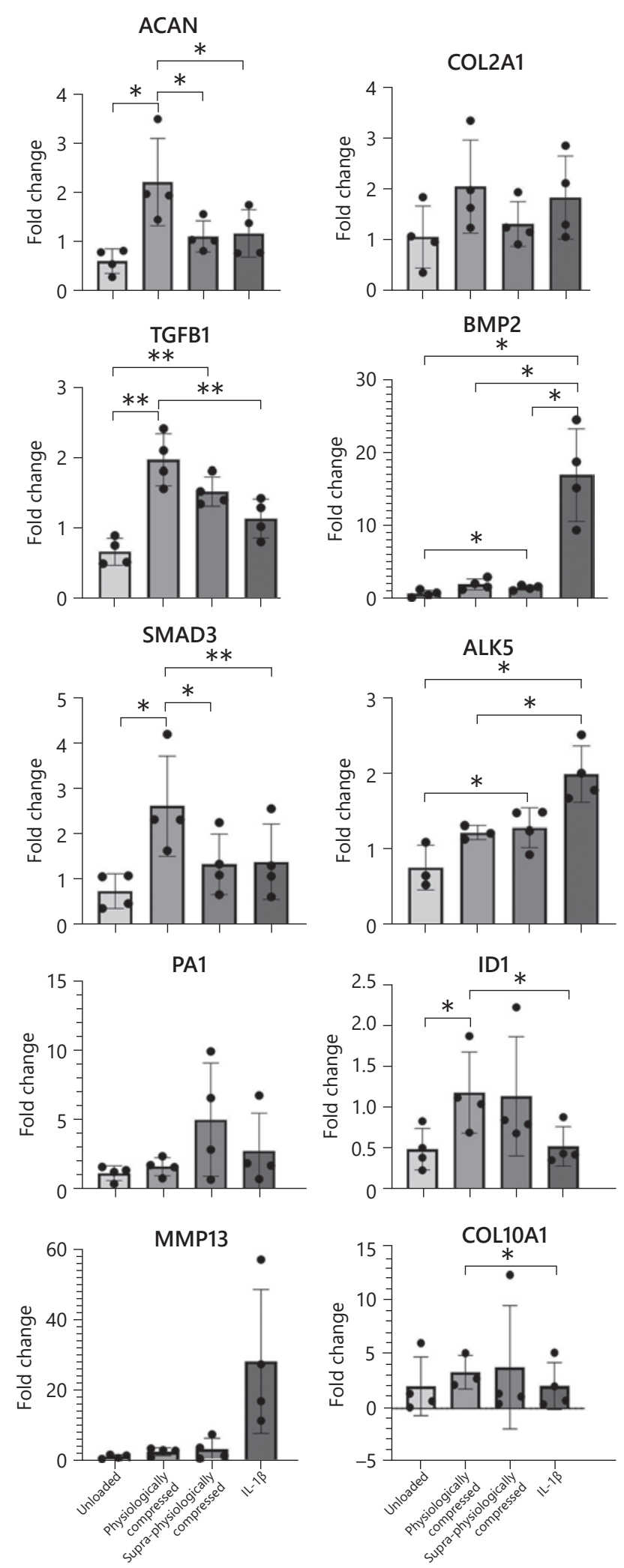


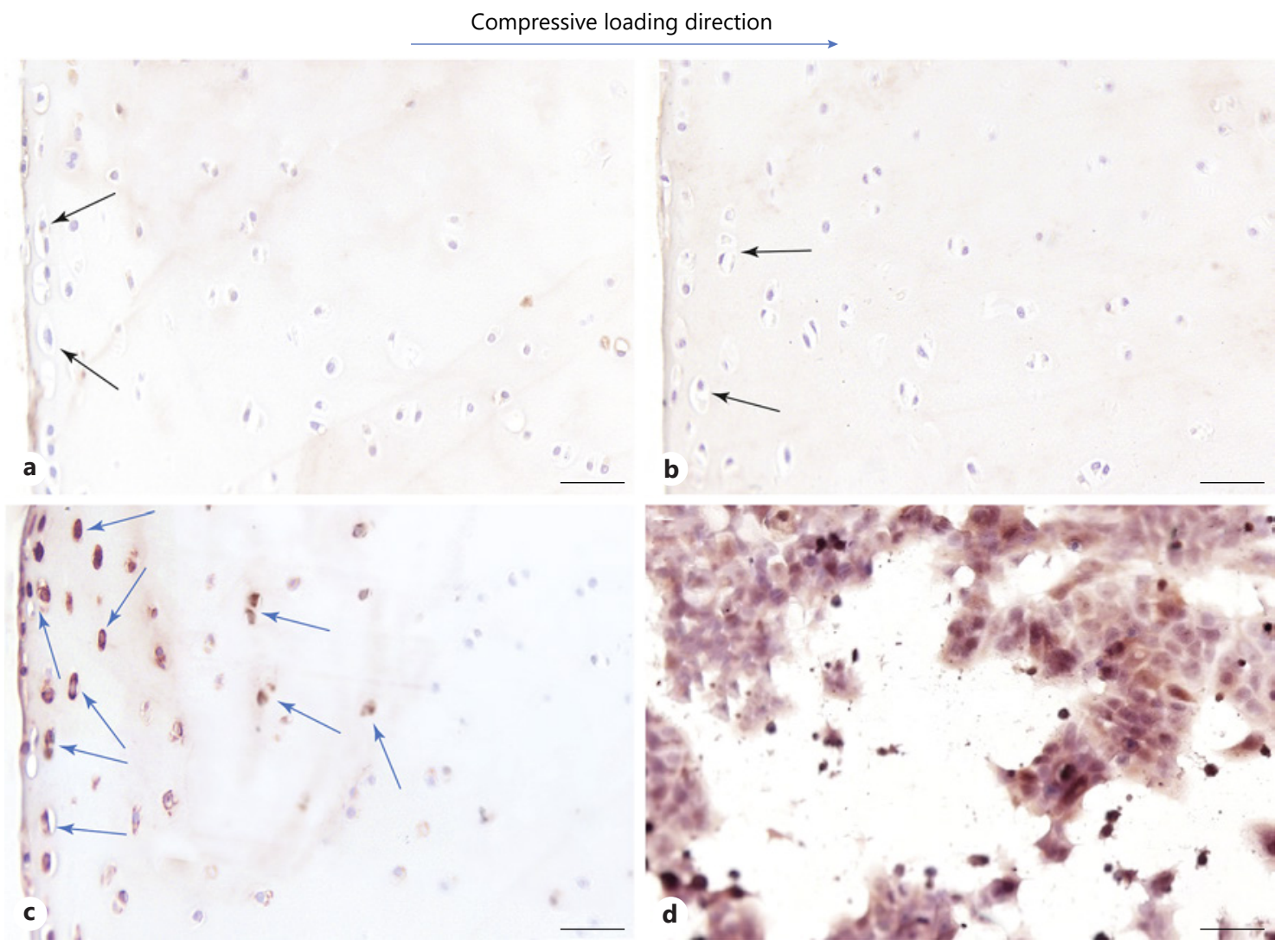

Fig. 7. Smad 3 immunohistochemistry on time zero group, before loading (a), unloaded group (b), loaded group (c), and positive immunostaining control for Smad 3 (MCF-7 cells) (d). Scale bar $=50 \mu \mathrm{m}$. Chondrocytes in time zero (a) and unloaded (b) samples do not show intracellular red staining, and only nuclei hematoxylin counterstain is visible (black arrows). Intracellular red staining is clearly noticeable in the superficial and intermediate zone (yellow arrows) of loaded sample (c) suggesting Smad 3 expression after mechanical activation.

were significantly higher, suggesting that the TGF- $\beta$ anabolic pathway was not activated. Finally, expression of $M M P 13$ showed an upward trend upon IL-1 $\beta$ treatment compared to all groups.

\section{Discussion}

Joint motion and weight-bearing are essential factors for maintaining articular cartilage health [Madej et al., 2016b]; however, both unloading and injurious loading affect tissue mechanobiological signaling and can lead to cartilage degeneration. In vitro studies are essential to allow analysis of the signaling cascades associated with different loading regimens by mimicking the conditions of daily normal and abnormal loads [Piscoya et al., 2005; Madej et al., 2016b; Issa et al., 2018]. A large number of studies have contributed to the understanding of the met- abolic and functional effects of trauma [Ulici et al., 2014; Madej et al., 2016a] with the aim to restore and/or preserve cartilage function as well as to develop in vitro models of OA for drug development and screening [Occhetta et al., 2019]. Furthermore, loading regimens have been proposed as approaches to drive stem cell phenotype in cartilage repair [Li et al., 2011; Iseki et al., 2019], as well as to develop in vitro engineered cartilage that more closely mimics native tissue [Luo et al., 2015; Salinas et al., 2018]. However, for all of these approaches, traditional one-sample-at-a-time methods are prohibitively timeconsuming, limiting the number of variables that can be effectively explored as well as the potential for clinical translation of experimental findings.

We report here the development of an automated mechanical activator device (HiT-MACE, Fig. 1a) as a highthroughput, user-friendly (Fig. 1b) system capable of individually loading up to 24 samples in one run. This allows 
to explore different compressive strains and loading regimens in each test, where the user can define specific parameters for a selected number of pistons, thus allowing to activate the 24 samples with different compressive regimes. Moreover, the device positively impacts on experimental time. Setting up the HiT-MACE sample amounts to only a few seconds under the hood to just place the explants in the multiwall plate and placing the lid on top. Then, 6 samples per donor for 4 donors (total of 24 samples) were mechanically activated for a half hour each, for a total time of $2 \mathrm{~h}$. The total time to perform the experiment from assembly to the processing step of the explants is $2.1 \mathrm{~h}$ at most. Using instead a single testing device, each individual explant assembly would take up to $5 \mathrm{~min}$ (by an optimistic estimate) followed by a half hour of activation for a total of $14 \mathrm{~h}$ for 24 explants. Compared to a single-sample system, the HiTMACE reduces by more than 6 times the time to perform this experiment, thus significantly lowering the cost of a multi-samples approach and rendering long-term studies more feasible. Furthermore, the custom-made lid apparatus ensures sterility during all procedures, which is essential for biological constructs (Fig. 1c). Additional advantages of this system include force sensing capabilities for each sample with individual point-of-contact control feedback, and experimental flexibility in changing compression parameters and selecting the samples subjected to load on a given plate. Such advanced features allow to perform experiments based on progressive compression loading using different displacement values between each cycle.

With this device, a user can sequentially test several 24-well plates, changing loading parameters and/or sample conditions, thereby rapidly generating a large sample size to investigate multiple experimental variables. Notably, to test this feature of the HiT-MACE, we tested 48 individual cartilage samples during two runs from 8 different porcine donors. As a result of this relatively high number, we were able to detect significant differences in gene expression even at an early time $(4.5 \mathrm{~h})$.

We validated the capacity of the HiT-MACE to precisely apply a defined mechanical deformation by measuring the modulus of different biomaterials (i.e., PDMS and agarose hydrogels of different concentrations) in a multi-well automated compression run and comparing these results with those obtained with a commercial compression tester ElectroForce ${ }^{\circledR} 3200$ Series III (by sampleby-sample measurement). We chose to use viscoelastic materials for this test since cartilage and many other engineered tissues are also viscoelastic.

According to the linear biphasic theory of cartilage, under low levels of strain $(<20 \%)$, the solid matrix follows the stress-strain law assumed for isotropic and linearly elastic materials [Guilak et al., 1994]. Thus, we applied compression at the same slow rate for both systems $(0.028 \mathrm{~Hz})$, and Hook's law was used to calculate the elastic modulus from the unloading curve to exclude any contribution from plastic deformation [Stolz et al., 2004]. The area between the loading and unloading curves is an indicator of the viscoelastic behavior of the tested materials: the large stressstrain hysteresis for agarose hydrogels suggests high energy dissipation during deformation and a dominant viscous component [Mohanraj et al., 2014a], whereas minimal hysteresis for PDMS suggests a viscoelastic-solid behavior [Johnston et al., 2014] (online suppl. Fig. S2). Overall, the moduli obtained with the HiT-MACE matched well with those obtained using the commercial compression tester, except for $4 \%$ and $6 \%$ agarose samples where the standard deviations were larger. This is not surprising, given the noise of the FSRs voltage amplitude output, resulting from a compromise between precision and cost containment. Notably, a one-way ANOVA with a post hoc Tukey test detects for both devices the same statistical differences between the moduli of each PDMS and agarose composition. Hence, both the commercial tester and the HiT-MACE are equally capable of distinguishing with good accuracy between samples even with relatively close values of modulus.

Unfortunately, this does not hold true when the lid engineered to maintain sterility is used (online suppl. Fig. S3). In fact, the piston-guide friction of the lid apparatus increases the contact stiffness as compared to the free slippage resulting in reduced accuracy and in an overestimate of the cartilage modulus [Cornelissen, 2013]. Controlling and modeling friction is challenging because of its nonlinearity [Széll et al., 2014] and is not the goal of this work. Our objective is to use the HiT-MACE to apply a user-controlled mechanical activation to full-thickness cartilage explants to explore tissue mechanobiological responses. Notably, if we maintain the same testing parameters, the friction for each piston-guide pair can be considered a constant offset within each experiment, and variations in loading can be reliably assessed. We have implemented this approach in the HiT-MACE control interface to identify the point of contact by an increase in load. Furthermore, our mechanical activator could similarly be used in long-term experiments to track changes in cartilage modulus due to ECM re-organization [Elder and Athanasiou, 2008], in response to injury, pro-inflammatory cytokines, regimens of mechanical activation, pharmaceutical candidates, etc.

In the current study, we performed a 30-min dynamic relaxation test to determine the deformation equilibrium 
response of the tissue over the range of physiological (15\% strain), slow-walking activity. We registered a significant increase in the compressive modulus after dynamic relaxation. This is consistent with previous studies that attribute the higher stiffness to cartilage viscoelastic behavior, i.e., to the decrease in hydration because of water exudation [Park et al., 2004; Han and Eriten, 2018]. As a consequence of this decrease in volume, proteoglycan density is increased and permeability is reduced, resulting in higher tissue resistance to deformation represented by a higher Young's modulus [Franz et al., 2001; Allen and Athanasiou, 2006]. Thus, variations in the modulus value after dynamic compression are expected and directly correlate with the change in hydration and rearrangement of the tissue matrix structure [Kumar et al., 2018]. The dynamic relaxation curve could not be fitted by a single exponential law which would be typical for the simple linear viscoelastic theory, which is not surprising given the biphasic nature of cartilage [Eisenfeld et al., 1978; Rubenstein et al., 1996]. Consequently, under the loading conditions we applied, our cartilage samples were characterized by two relaxation times: a faster time related to fluid outflow, and a slower relaxation response due to changes in proteoglycan concentration and spatial distribution [Bansalyz et al., 2010; Thambyah and Broom, 2013]. Furthermore, we obtained a close-to-zero equilibrium stress, consistent with a cartilage relaxation response mostly viscous, as described by Thambyah and Broom [2013], as a result of the matrix swelling pressure generated by the increase in proteoglycan concentration at low strains. Previous studies have shown how during OA progression, cartilage experiences a decrease in relaxation time (i.e., a decrease in GAG content), equilibrium modulus, and stiffness [June et al., 2009; Kumar et al., 2018], whereas dynamic, physiological compression promotes an improvement in ECM organization and synthesis [Darling and Athanasiou, 2003]. Hence, our system could in principle be used in long-term studies to monitor changes in modulus and relaxation of in vitro OA models as well as to deliver pro-anabolic mechanical stimuli.

After validating our device by testing viscoelastic materials and native cartilage, HiT-MACE was used as a high-throughput activator to investigate how different amplitudes of dynamic compression direct the shortterm anabolic or catabolic response of cartilage explants. It has been widely reported that physiological compression promotes a pro-anabolic phenotype in native and engineered cartilage [Guilak et al., 1994; Park et al., 2004; Iseki et al., 2019], whereas supra-physiological loading has been proposed as a realistic approach to model OA and initiate matrix degradation [Piscoya et al., 2005; Oc-

Mechanical Activator for in vitro Response of Cartilage to Loading chetta et al., 2019]. Hence, we first compared the effects of a short-term (30 $\mathrm{min})$ physiological $(1 \mathrm{~Hz}, 20 \%$ strain) or supra-physiological $(1 \mathrm{~Hz}, 50 \%$ strain) loading on the transcriptional response of articular cartilage. Then, we assessed how the response to supra-physiological loading differs from induction of catabolism by the pro-inflammatory cytokine IL- $1 \beta$, which has frequently been adopted as a biochemical inducer of $\mathrm{OA}$ in in vitro model.

For research using porcine cartilage, RPL4 and GAP$D H$ are the most commonly used housekeeping genes. However, several studies have shown that mechanical activation influence their CT value and their stability in expression [Nygard et al., 2007; Al-Sabah et al., 2016], in particular after a compression corresponding to a $10 \%$ strain. The same authors identified $\beta$-actin, ACTB, as the gene with the least affected transcription level either by cyclic physiological loading, than by higher levels of stress ( $8 \mathrm{MPa}, 1 \mathrm{~Hz}, 15$ minutes) [Al-Sabah et al., 2016]. Hence, we chose $A C T B$ as the most suitable housekeeping gene to normalize our gene expression analysis for our dynamic mechanobiological study.

In studying cartilage response to loading, we first sought to determine whether a relatively brief physiological compressive loading (half hour) activates the anabolic TGF- $\beta$ signaling, an important pathway in promoting the expression of pro-anabolic Smad2/3 dependent cartilage genes [Santibañez et al., 2011; Choi et al., 2017]. On the other hand, the TGF- $\beta$ mediated Smad $1 / 5 / 8$ activation promotes a more catabolic response in chondrocytes metabolism leading to hypertrophy and terminal differentiation [Mariani et al., 2014; Kraan, 2018]. When we subjected cartilage to physiological loading, our results showed an upregulation of TGFB1 as well as ALK5 and SMAD3, along with an increase in the expression of downstream factors associated with the TGF- $\beta / \mathrm{Smad} 2 / 3$ pathway, such as COL $2 A 1$ and ACAN (Fig. 5). The expression of PAI1 (serpine1), a gene downstream of the $S m a d 2 / 3$ pathway, did not change significantly, consistent with the findings of Madej et al. [2014] under similar experimental conditions. Thus, we focused primarily on ACAN and COL2 as Smad 3 activated genes. Overall, our findings suggest that even a short, 30min dynamic physiological loading (15\% strain) activates genes whose function is to enhance matrix synthesis, thus suggesting inhibition of early chondrocyte terminal differentiation, similarly to what was previously reported by others [Santibañez et al., 2011; Sanchez-Adams et al., 2014; Madej et al., 2016b; Schätti et al., 2016]. As shown in Figure 7 , immunohistochemistry showed that the controls did not stain for Smad3 (Fig. 7a, b), whereas chondrocytes undergoing physiological loading were strongly positive (Fig. 7c). 
Remarkably, Smad3 protein was more highly expressed in the superficial zone, the area more directly exposed to mechanical activation. The staining was gradually less evident moving towards the intermediate zone, and completely disappeared in the deep zone.

As for the other genes, ID1 is a key transcription factor commonly used as a specific marker of the Smad 1/5/8 signaling route [Madej et al., 2014]; hence, its expression was analyzed to monitor the pro-catabolic Smad pathway. Although in general BMP proteins induce receptorregulated Smad1/5/8 activation (Fig. 4), BMP2 specifically is well known to be associated with ECM production and maintenance, i.e., a matrix rich in sulfated glycosaminoglycans and collagen type II, resulting in cartilage formation and enhancing tissue homeostasis [Kopf et al., 2012; Van Caam et al., 2016; Anderson and Johnstone, 2017; Oladazimi et al., 2019]. Here we observed upregulation of both BMP2 and ID1 upon loading, which could appear contradictory. Yet, it has been reported that Id1 transcription level is induce early by BMP- 2 and TGF- $\beta 1$ activation, with Smad3 mediating this regulation while binding the native Id1 promoter [Liang et al., 2009]. Moreover, Kopf et al. [2012] showed that an early Id1 induction from BMP2 activation is significantly enhanced when cells are concurrently mechanically loaded.

Overall, we reported that physiological mechanical loading promotes an anabolic response in chondrocytes metabolism. Notably, we were able to obtain statistically significant results in a rapid manner with the HiT-MACE system. Indeed, we compared data from 8 porcine donors just running our custom-made device twice (once each day of experiment), with the rate limiting step being sample extraction rather than mechanical activation. Thus, we confirmed the throughput potential of our system and how it can contribute to the study of cartilage mechanobiology.

Traumatic injury, overloading, and inflammatory-induced cellular responses are the main causes of cartilage ECM damage and degradation, leading to the initiation and progression of OA [Blain et al., 2001; Tanne, 2008; Párraga Quiroga et al., 2017]. In experimental in vitro models of OA, the addition of IL- $1 \beta$ [Stevens et al., 2009] in culture medium is the most common and simple method used to simulate the inflammatory environment associated with OA [Xie et al., 2015]. We have previously shown that the introduction of IL- $1 \beta$ to either the chondral or osseous medium stream of a biphasic bioreactor induced strong degradative responses in human stem cell-derived osteochondral constructs [Lin et al., 2014]. However, in vivo models of disease derived by the administration of exogenous IL- $1 \beta$ do not reflect the spontane- ous changes occurring in OA development, but rather alterations following a rapid and acute inflammatory response [Thysen et al., 2015]. Hence, IL- $1 \beta$ treatment simulates only a very narrow part of the more complex mechanisms that are activated in OA. Recently, Occhetta et al. [2019] focused on supra-physiological dynamic compression as a potentially more biomimetic inducer of OA [Gottardi, 2019], using a cartilage-on-a-chip device that combines microfluidics and controlled loading of primary chondrocytes in a PEG hydrogel. They compared the response to 7 days of supra-physiological loading versus exposure to IL- $1 \beta$ and found that the pro-catabolic response induced by loading better matches the profile observed in vivo in humans. While potentially high throughput, the microfluidic cartilage-on-chip device used by Occhetta et al. [2019] can only work with engineered constructs. HiT-MaCE, on the other hand, can also work with native tissues. Thus, taking advantage of the throughput potential of our system, we compared supra-physiological loading and IL- $1 \beta$ exposure, focusing on the early response of porcine cartilage explants.

Specifically, we found that a short exposure to IL-1 $\beta$ already elicited the expression of catabolic mediators typical of OA [Santibañez et al., 2011; Torzilli et al., 2011; Sanchez-Adams et al., 2014], whereas a supra-physiological loading did not induce a significant shift towards catabolism. This is not surprising, given the similar observations made by Madej et al. [2014], suggesting that a longer timeframe is required for supraphysiological loading models of $\mathrm{OA}$ induction.

We observed that $A L K 5$ was significantly upregulated by both physiological (Fig. 5, $n=8$ donors) and supra-physiological (Fig. 6, $n=4$ donors) loading, reaching significance faster for the latter. Treatment with IL- $1 \beta$ also rapidly upregulates $A L K 5$ (Fig. 6), suggesting potential Smad $2 / 3$ activation for all groups. Nevertheless, our analysis of Alk5downstream anabolic genes shows that SMAD3 and ACAN are significantly upregulated only after physiological compression, but not in the other conditions (Fig. 6).

Remarkably, prior studies have noted that Alk5 is involved in both anabolism and at the onset of catabolic responses. In fact, Oladazimi et al. [2019] reported the overexpression of multiple genes, including $A L K 5$, in an $\mathrm{OA}$ destabilization model in rats, and Alk5 kinase activity has been shown to be crucial to induce Smad1/5 signaling [Van Caam et al., 2017]. The latter is a driver of hypertrophic differentiation of chondrocytes and may promote cartilage degradation by increasing MMP13 expression [Wang et al., 2014], generally associated with cartilage hypertrophy [Eberli, 2011; Van Caam et al., 2016; Anderson and Johnstone, 
2017]. Similarly, Mariani et al. [2014] described how BMP2 plays a role in matrix degradation in OA by stimulating the production of MMP13 via SMAD1/5/8 phosphorylation. The RT-PCR results from our IL- $1 \beta$ stimulated samples showed an increasing trend for $M M P 13$ expression $(p=$ $0.07, n=4$ ), a significant upregulation for both $B M P 2$ and $A L K 5$, but no changes in TGFB1 expression nor its downstream anabolic markers SMAD3, ACAN, COL2, PAI1. Taken together, these findings suggest the activation of the Smad1/5/8 to MMP13 signaling pathway and a rapid shift towards matrix degradation in the pro-inflammatory group. Downstream of BMP2 and Smad 1/5/8 is also the gene COL10, a hallmark of late-stage chondrocyte hypertrophy, for which we observed no change. This is not surprising as COL10 is a late-expression gene that was unlikely to be induced with such a short IL- $1 \beta$ treatment (or mechanical activation, for that matter) [Luo et al., 2017].

Our data show that neither physiological nor supraphysiological compression activates MMP13 expression, which suggests that chondrocytes may respond to different levels of dynamic loading without being affected to assume a hypertrophic phenotype, at least in the early response time frame. Similar findings were reported by Patwari et al. [2003], who studied the effects of short-term, injurious mechanical compression in cartilage explants. They applied a ramp to $50 \%$ strain at a $1-\mathrm{Hz}$ rate, followed by release, and observed no change in MMP13 in the first $24 \mathrm{~h}$, evidencing how overloading alone did not activate matrix degradation in the short term, unless IL- $1 \beta$ was also present [Patwari et al., 2003]. However, the absence of degradative factors in cartilage injury models that use supra-physiological mechanical activation, such as ours and Patwari's, might be somewhat surprising.

In fact, Occhetta et al. [2019] found a significant increase in MMP13 expression after 7 days of intermittent supraphysiological dynamic compression ( $30 \%$ strain, $1 \mathrm{~Hz}$, with $2 \mathrm{~h}$ stimulation, $4 \mathrm{~h}$ rest, $2 \mathrm{~h}$ stimulation, and $16 \mathrm{~h}$ rest per day) of primary chondrocytes in a PEG hydrogel. Moreover, they observed a reduction in the expression of the anabolic genes COL2 and ACAN and the onset of a hypertrophic cartilage phenotype marked by the increased expression levels of COL10. Overall, these findings are markedly dissimilar from those seen in the present study, which might be attributed to the differences in mechanical transduction between native cartilage matrix and a PEG hydrogel or, more likely, to the effects on cellular pathways of short versus long-term application of dynamic loading. In fact, Fitzgerald et al. [2004] applied a 50\% static compression on cartilage explants and observed how MMP13 expression does not change after $1,2,4$, or $8 \mathrm{~h}$, but increases

Mechanical Activator for in vitro

Response of Cartilage to Loading by 8 -fold by $24 \mathrm{~h}$. Their work suggests that $M M P 13$ does not change immediately after compression, as we also observed in our work, but becomes evident over a longer time course as reported by Occhetta et al. [2019]. Indeed, cellular responses to mechanical stress involve multiple mechanotransduction pathways that modulate gene expression and cartilage remodeling in a time-dependent manner.

To better understand the possible effects of our overloading protocol, we compared the changes in gene expression we found for the supra-physiological group to those of the physiological and pro-inflammatory groups, which clearly resulted in pro-anabolic and pro-catabolic responses, respectively. Cartilage discs subjected to $50 \%$ strain revealed an imbalance between anabolic and catabolic activity, with a significant increase in TGFB1, BMP2, and $A L K 5$, but only a positive trend in $A C A N, I D 1$, and $S M A D 3$, and no changes in COL2A1, COL10, and MMP13. The most prominent anabolic effect is the upregulation of TGFB1 that may result in endogenous TGF- $\beta 1$ production, i.e., a central counteracting factor of cartilage degradation and of OA development [Takahashi et al., 2005]. At the same time, COL2A1 did not change, and the increase in expression of SMAD3 and ACAN as well as of ID1 (whose early induction requires Smad3 binding, along with TGFB1 and/or BMP2 activation [Liang et al., 2009; Kopf et al., 2012]) were only trending but not statistically significant. These results show an overall weaker anabolic response after supra-physiological loading compared to the physiological activation. At the same time, while activation of pro-catabolic markers such as MMP13 was not observed, it could still rise after a day or more from overloading in accordance with the works of Fitzgerald et al. [2004] and Occhetta et al. [2019].

In this respect, $B M P 2$ and $A L K 5$ were also significantly upregulated in supra-physiological loading, although not to the same extent as the IL- $1 \beta$ group. As previously discussed, the BMP2 growth factor and ALK5 receptor may play a significant role either in maintaining cartilage phenotype or in modulating its inflammatory reaction. When the latter activity occurs, it leads to an increased MMP13 expression, which we observed only in the IL- $1 \beta$ group.

It is then possible that the initial response to supraphysiologically mechanical activation is one of a mild repair/protective Smad3 pathway activation to inhibit a pro-catabolic response that may further damage cartilage. Therefore, we could hypothesize that over a longer time course, the supra-physiological loading would induce a severely injurious response in articular cartilage, i.e., the onset of matrix degradation and hypertrophic chondrocyte phenotype, caused by a progressive imbal- 
ance of anabolic/catabolic tissue remodeling towards catabolism.

Such hypothesis can be enforced studying the response of cartilage, or even better of bone-cartilage composite tissue, to a combined compression-shear mechanical stress. In fact, the most relevant issues of this work are linked to some limitations of our model that could be further improved. First, our loading protocol was a simple compression, which differs from the more complex combination of compression and shear stress in vivo. In addition, we have only examined cartilage explants rather than osteochondral units, which did not allow the analysis of cartilage-bone interactions in the development of OA.

In future studies, we plan to examine the influence of different loading regimes at multiple time points to investigate the sequential nature of mechanotransduction signaling over time. Moreover, we will study the effects of combined compression and IL- $1 \beta$ exposure, and of specific combinations and sequences of physiological supraphysiological loading, to explore how loading regimens, such as that during rehabilitation, could be used to mitigate cartilage damage caused by inflammation and overloading.

\section{Conclusions}

We have engineered a compressive mechanical activator device (HiT-MACE) that can stimulate up to 24 samples in one run, thus creating a system that allows to perform experiments over 6 times faster than a single testing device. The HiT-MACE was first validated using synthetic and natural materials (PDMS and agarose) and cartilage, showing good accuracy in (1) delivering mechanical stimulations, (2) measuring Young's modulus of biomaterials, and (3) detecting the viscoelastic behavior of cartilage tissue following a dynamic relaxation. However, for use with biological samples, a lid apparatus is required to maintain sterility which impairs determination of the Young's modulus of cartilage explants during mechanical activation. Nevertheless, controlled mechanical activation to investigate mechanobiological responses of chondrocytes in a $3 \mathrm{D}$ tissue context was successfully performed simultaneously for 4 different donors in only $2 \mathrm{~h}$, confirming the potential of HiT-MACE for faster and higher throughput experiments. Specifically, using HiTMACE, we have characterized the early biomechanical response of porcine articular cartilage explants following regimens of physiological and supra-physiological dy- namic compression, as well as an acute IL- $1 \beta$ treatment. Gene expression profiling revealed that physiological loading rapidly activates markers of ECM synthesis and tissue homeostasis via the anabolic TGF- $\beta / S \operatorname{Smad} 3$ pathway, whereas supra-physiological compression induces an initial remodeling response that might delay the onset of tissue degeneration. In contrast, stimulation by IL- $1 \beta$ showed a rapid enhancement MMP13 expression and markers associated with OA, suggesting an acute pro-inflammatory response.

\section{Acknowledgments}

The authors would like to acknowledge Dr. Douglas A. Nelson Jr. and Mr. Dan McKeel at the University of Pittsburgh, PA, for their help and contribution to the design and manufacturing of the HiT-MACE device.

\section{Statement of Ethics}

The paper is exempt from ethical committee approval as the cartilage plugs used in this work were harvested from knee joints obtained from a local abattoir.

\section{Conflict of Interest Statement}

The authors have no conflicts of interest to declare.

\section{Funding Sources}

This work was supported in part by Ri.MED Foundation (Italy), the U.S. Department of Defense (W81XWH-08-2-0032 and W81XWH-10-1-0850), the National Institutes of Health (NCATS U18TR000532), the Children's Hospital of Philadelphia Research Institute, the Frontier Program in Airway Disorders of the Children's Hospital of Philadelphia, and the European Union under the Horizon 2020 Framework Research Program SC1-PM17-2017 - Project OACTIVE - Grant Agreement 777159.

\section{Author Contributions}

R.G., V.L.C., V.B., and R.S.T. conceptualized and designed the work; E.C. performed the experiments on biological tissues and analyzed the data with R.D.G., V.L.C., and R.G.; D.M., V.B., and R.G. set up and tested the activation system on gels and analyzed the results; E.C., R.D.G., R.S.T., and R.G. drafted and revised critically the manuscript. All authors read and approved the manuscript. 


\section{References}

Alexander PG, Song Y, Taboas JM, Chen FH, Melvin GM, Manner PA, et al. Development of a Spring-Loaded Impact Device to Deliver Injurious Mechanical Impacts to the Articular Cartilage Surface. Cartilage. 2013;4(1):52-62.

Allen KD, Athanasiou KA. Viscoelastic characterization of the porcine temporomandibular joint disc under unconfined compression. J Biomech. 2006;39(2):312-22.

Al-Sabah A, Stadnik P, Gilbert SJ, Duance VC, Blain EJ. Importance of reference gene selection for articular cartilage mechanobiology studies. Osteoarthr Cartil. 2016;24(4):719-30.

Anderson DE, Johnstone B. Dynamic Mechanical Compression of Chondrocytes for Tissue Engineering: A Critical Review. Front Bioeng Biotechnol. 2017;5:76.

Arokoski JP, Jurvelin JS, Väätäinen U, Helminen HJ. Normal and pathological adaptations of articular cartilage to joint loading. Scand J Med Sci Sport. 2000;10:186-98.

Bansalyz PN, Joshix NS, Entezariy V, Grinstaffzx MW, Snydery BD. Contrast Enhanced Computed Tomography can predict the glycosaminoglycan content and biomechanical properties of articular cartilage. Osteoarthr Cartil. 2010;18:184-91.

Blain EJ, Gilbert SJ, Wardale RJ, Capper SJ, Mason DJ, Duance VC. Up-regulation of matrix metalloproteinase expression and activation following cyclical compressive loading of articular cartilage in vitro. Arch Biochem Biophys. 2001;396(1):49-55.

Bonnevie ED, Delco ML, Fortier LA, Alexander PG, Tuan RS, Bonassar LJ. Characterization of tissue response to impact loads delivered using a hand-held instrument for studying articular cartilage injury. Cartilage. 2015;6(4): 226-32.

Carbone A, Rodeo S. Review of current understanding of post-traumatic osteoarthritis resulting from sports injuries. J Orthop Res. 2017;35(3):397-405.

Cespi M, Bonacucina G, Misici-Falzi M, Golzi R, Boltri L, Palmieri GF. Stress relaxation test for the characterization of the viscoelasticity of pellets. Eur J Pharm Biopharm. 2007;67(2): 476-84.

Ru Choi and Wey Yong, 2017 Choi JR, Yong KW Choi JY. Effects of mechanical loading on human mesenchymal stem cells for cartilage tissue engineering. J Cell Physiol. 2018 Mar 233(3):1913-28.

Cohen NP, Foster RJ, Mow VC. Composition and Dynamics of Articular Cartilage: Structure, Function, and Maintaining healthy State. J Orthop Sports Phys Ther. 1998;28(4):203-15.

Cornelissen B. The role of friction in tow mechanics. Enschede: University of Twente, 2013.

Darling EM, Athanasiou KA. Biomechanical Strategies for Articular Cartilage Regeneration. Ann Biomed Eng. 2003;31(9):1114-24.

De Croos JN, Dhaliwal SS, Grynpas MD, Pilliar RM, Kandel RA. Cyclic compressive mechanical stimulation induces sequential catabolic and anabolic gene changes in chondrocytes resulting in increased extracellular matrix accumulation. Matrix Biol. 2006;25(6):323-31.

De Lise AM, Fischer L, Tuan RS. Cellular interactions and signaling in cartilage development. Osteoarthr Cartil. 2000;8:309-34.

Di Federico E, Bader DL, Shelton JC. Design and validation of an in vitro loading system for the combined application of cyclic compression and shear to $3 \mathrm{D}$ chondrocytes-seeded agarose constructs. Med Eng Phys. 2014;36(4):53440.

Eberli D. Tissue engineering for tissue and organ regeneration. 2011.

Eisenfeld J, Mow VC, Lipshitz H. Mathematical analysis of stress relaxation in articular cartilage during compression. Mathematical Biosciences. 1978;39(1凶2):97-112.

Elder BD, Athanasiou KA. Effects of Confinement on the Mechanical Properties of SelfAssembled Articular Cartilage Constructs in the Direction Orthogonal to the Confinement Surface. J Orthop Res. 2008;26(2):238-46.

Fitzgerald JB, Jin M, Chai DH, Siparsky P, Fanning P, Grodzinsky AJ. Shear- and compression-induced chondrocyte transcription requires MAPK activation in cartilage explants. J Biol Chem. 2008;283(11):6735-43.

Fitzgerald JB, Jin M, Dean D, Wood DJ, Zheng $\mathrm{MH}$, Grodzinsky AJ. Mechanical compression of cartilage explants induces multiple time-dependent gene expression patterns and involves intracellular calcium and cyclic AMP. J Biol Chem. 2004;279(19):19502-11.

Frank EH, Jin M, Loening AM, Levenston ME, Grodzinsky AJ. A versatile shear and compression apparatus for mechanical stimulation of tissue culture explants. J Biomech. 2000;33(11):1523-7

Franz T, Hasler EM, Hagg R, Weiler C, Jakob RP, Mainil-Varlet P. In situ compressive stiffness, biochemical composition, and structural integrity of articular cartilage of the human knee joint. Osteoarthr Cartil. 2001;9(6):58292.

Gottardi R. Load-induced osteoarthritis on a chip. Nat Biomed Eng. 2019;3(7):502-3.

Gottardi R, Stoddart MJ. Regenerative Rehabilitation of the Musculoskeletal System. J Am Acad Orthop Surg. 2018;26(15):e321-3.

Guilak F, Meyer BC, Ratcliffe A, Mow VC. The effects of matrix compression on proteoglycan metabolism in articular cartilage explants. Osteoarthr Cartil. 1994;2(2):91-101.

Han G, Eriten M. Effect of relaxation-dependent adhesion on pre-sliding response of cartilage. R Soc Open Sci. 2018;5.

Iseki T, Rothrauff BB, Kihara S, Sasaki H, Yoshiya S, Fu FH, et al. Dynamic Compressive Loading Improves Cartilage Repair in an In Vitro Model of Microfracture: Comparison of 2 Mechanical Loading Regimens on Simulated Microfracture Based on Fibrin Gel Scaffolds Encapsulating Connective Tissue Progenitor Cells. Am J Sports Med. 2019;47(9):2188-99.
Issa R, Boeving M, Kinter M, Griffin TM. Effect of biomechanical stress on endogenous antioxidant networks in bovine articular cartilage. J Orthop Res. 2018;36(2):760-9.

Johnston ID, McCluskey DK, Tan CKL, Tracey MC. Mechanical characterization of bulk Sylgard 184 for microfluidics and microengineering. J Micromech Microeng. 2014;24(3): 035017.

June RK, Ly S, Fyhrie DP. Cartilage stress-relaxation proceeds slower at higher compressive strains. Arch Biochem Biophys. 2009;483(1): 75-80.RK

Kopf J, Petersen A, Duda GN, Knaus P. BMP2 and mechanical loading cooperatively regulate immediate early signalling events in the BMP pathway. BMC Biol. 2012;10:37.

Kumar R, Pierce DM, Isaksen V, Davies CDL, Drogset JO, Lilledahl MB. Comparison of compressive stress-relaxation behavior in osteoarthritic (ICRS graded) human articular cartilage. Int J Mol Sci. 2018;19.

Lawless BM, Sadeghi H, Temple DK, Dhaliwal H, Espino DM, Hukins DWL. Viscoelasticity of articular cartilage: Analysing the effect of induced stress and the restraint of bone in a dynamic environment. J Mech Behav Biomed Mater. 2017;75:293-301.

Li LP, Buschmann MD, Shirazi-Adl A. Strain-rate Dependent Stiffness of Articular Cartilage in Unconfined Compression. J Biomech Eng. 2003;125(2):161-8

Li NG, Shi ZH, Tang YP, Wang ZJ, Song SL, Qian $\mathrm{LH}$, et al. New Hope for the Treatment of Osteoarthritis Through Selective Inhibition of MMP-13. Curr Med Chem. 2011;18(7):9771001.

Liang YY, Brunicardi FC, Lin X. Smad3 mediates immediate early induction of Id1 by TGF-beta. Cell Res. 2009;19(1):140-8.

Lin H, Lozito TP, Alexander PG, Gottardi R, Tuan RS. Stem Cell-Based Microphysiological Osteochondral System to Model Tissue Response to Interleukin-1 $\beta$. Mol Pharm.2014 Jul 7;11(7):2203-12.

Lujan TJ, Wirtz KM, Bahney CS, Madey SM, Johnstone B, Bottlang M. A novel bioreactor for the dynamic stimulation and mechanical evaluation of multiple tissue-engineered constructs. Tissue Eng Part C Methods. 2011; 17(3):367-74.

Luo L, Thorpe SD, Buckley CT, Kelly DJ. The effects of dynamic compression on the development of cartilage grafts engineered using bone marrow and infrapatellar fat pad derived stem cells. Biomed Mater. 2015;10(5):055011.

Luo Y, Sinkeviciute D, He Y, Karsdal M, Henrotin $\mathrm{Y}$, Mobasheri A, et al. The minor collagens in articular cartilage. Protein Cell. 2017;8(8): 560-72.

Madej W, Buma P, Van der Kraan P. Inflammatory conditions partly impair the mechanically mediated activation of Smad2/3 signaling in articular cartilage. Arthritis Res Ther. 2016a;18:146. 
Madej W, Van Caam A, Blaney Davidson E, Buma P, Van der Kraan PM. Unloading results in rapid loss of TGF $\beta$ signaling in articular cartilage: role of loading-induced TGF $\beta$ signaling in maintenance of articular chondrocyte phenotype? Osteoarthr Cartil. 2016b; 24:1807-15.

Madej W, Van Caam A, Blaney Davidson EN, van der Kraan PM, Buma P. Physiological and excessive mechanical compression of articular cartilage activates Smad2/3P signaling. Osteoarthr Cartil. 2014;22(7):1018-25.

Mariani E, Pulsatelli L, Facchini A. Signaling pathways in cartilage repair. Int J Mol Sci. 2014;15(5):8667-98.

Marklund E, Eitzenberger J, Varna J. Nonlinear viscoelastic viscoplastic material model including stiffness degradation for hemp/lignin composites. Composites Science and Technology. 2008;68(9):2156-62.

Mauck RL, Byers BA, Yuan X, Tuan RS. Regulation of cartilaginous ECM gene transcription by chondrocytes and MSCs in 3D culture in response to dynamic loading. Biomech Model Mechanobiol. 2007;6(1囚2):113-25.

Mohanraj B, Hou C, Meloni GR, Cosgrove BD, Dodge GR, Mauck RL. A high throughput mechanical screening device for cartilage tissue engineering. J Biomech. 2014a;47(9): 2130-6.

Mohanraj B, Meloni GR, Mauck RL, Dodge GR. A high-throughput model of post-traumatic osteoarthritis using engineered cartilage tissue analogs. Osteoarthr Cartil. 2014b;22(9): 1282-90.

Mueller MB, Tuan RS. Functional characterization of hypertrophy in chondrogenesis of human mesenchymal stem cells. Arthritis Rheum. 2008;58(5):1377-88.

Nygard AB, Jørgensen CB, Cirera S, Fredholm M. Selection of reference genes for gene expression studies in pig tissues using SYBR green qPCR. BMC Mol Biol. 2007;8:67.

Occhetta P, Mainardi A, Votta E, Vallmajo-Martin Q, Ehrbar M, Martin I, et al. Hyperphysiological compression of articular cartilage induces an osteoarthritic phenotype in a cartilage-on-a-chip model. Nat Biomed Eng. 2019; 3(7):545-57.

Oladazimi S, Farzanegi P, Azarbayejani MA. Evaluation of ALK5 and MMP13 Expression in the Cartilage Tissue of Rats with Osteoarthritis Rats and Effects of Exercise Training, Ozone and Mesenchymal Stem Cell Therapies on Expression of these Genes. Med Lab J. 2019;14:50-6.

Park S, Hung CT, Ateshian GA. Mechanical response of bovine articular cartilage under dynamic unconfined compression loading at physiological stress levels. Osteoarthr Cartil. 2004;12(1):65-73.

Párraga Quiroga JM, Wilson W, Ito K, Van Donkelaar CC. The effect of loading rate on the development of early damage in articular cartilage. Biomech Model Mechanobiol. 2017; 16(1):263-73.
Patwari P, Cook MN, DiMicco MA, Blake SM, James IE, Kumar S, et al. Proteoglycan degradation after injurious compression of bovine and human articular cartilage in vitro: Interaction with exogenous cytokines. Arthritis Rheum. 2003;48(5):1292-301.

Piscoya JL, Fermor B, Kraus VB, Stabler TV, Guilak F. The influence of mechanical compression on the induction of osteoarthritisrelated biomarkers in articular cartilage explants. Osteoarthr Cartil. 2005;13(12):1092-

Praxenthaler H, Krämer E, Weisser M, Hecht N, Fischer J, Grossner T, et al. Extracellular matrix content and WNT/ $\beta$-catenin levels of cartilage determine the chondrocyte response to compressive load. Biochim Biophys Acta Mol Basis Dis. 2018;1864(3):851-9.

Rubenstein JD, Kim JK, Henkelman RM. Effects of Compression and Recovery on Bovine Articular Cartilage: Appearance on MR Images. Radiology. 1996;201(3):843-50.

Salinas EY, Hu JC, Athanasiou K. A Guide for Using Mechanical Stimulation to Enhance Tissue-Engineered Articular Cartilage Properties. Tissue Eng Part B Rev. 2018. ten. teb.2018.0006.

Sanchez-Adams J, Leddy HA, McNulty AL, O'Conor CJ, Guilak F. The mechanobiology of articular cartilage: bearing the burden of osteoarthritis. Curr Rheumatol Rep. 2014; 16(10):451

Santibañez JF, Quintanilla M, Bernabeu C. TGF- $\beta /$ TGF- $\beta$ receptor system and its role in physiological and pathological conditions. Clin Sci. 2011;121(6):233-51.

Sauerland K, Raiss RX, Steinmeyer J. Proteoglycan metabolism and viability of articular cartilage explants as modulated by the frequency of intermittent loading. Osteoarthr Cartil. 2003;11(5):343-50.

Schatti O, Grad S, Goldhahn J, Salzmann G, Li Z, Alini $M$, et al. A combination of shear and dynamic compression leads to mechanically induced chondrogenesis of human mesenchymal stem cells. Eur Cell Mater. 2011;22:21425.

Schätti OR, Gallo LM, Torzilli PA. A Model to Study Articular Cartilage Mechanical and Biological Responses to Sliding Loads. Ann Biomed Eng. 2016;44(8):2577-88.

Stevens AL, Wishnok JS, White FM, Grodzinsky AJ, Tannenbaum SR. Mechanical Injury and Cytokines Cause Loss of Cartilage Integrity and Upregulate Proteins Associated with $\mathrm{Ca}$ tabolism, Immunity, Inflammation, and Repair. Mol Cell Proteomics. 2009;8(7):147589.

Stolz M, Raiteri R, Daniels AU, VanLandingham MR, Baschong W, Aebi U. Dynamic elastic modulus of porcine articular cartilage determined at two different levels of tissue organization by indentation-type atomic force microscopy. Biophys J. 2004;86(5):3269-83.
Széll K, Czmerk A, Korondi P. Friction with Hysteresis Loop Modeled by Tensor Product. Automatika. 2014;55(4):463-73.

Taffetani M, Bertarelli E, Gottardi R, Raiteri R, Vena P. Modelling of the frequency response to dynamic nanoindentation of soft hydrated anisotropic materials: Application to articular cartilage. C - Comput Model Eng Sci. 2012;87: 433-60.

Takahashi N, Rieneck K, Van der Kraan PM, Van Beuningen HM, Vitters EL, Bendtzen K, et al. Elucidation of IL-1/TGF-beta interactions in mouse chondrocyte cell line by genome-wide gene expression. Osteoarthr Cartil. 2005; 13(5):426-38.

Tanne K. Degenerative changes of articular cartilage in association with mechanical stimuli. Japanese Dental Science Review. 2008;44(1): 38-47.

Thambyah A, Broom ND. Further insight into the depth-dependent microstructural response of cartilage to compression using a channel indentation technique. Comput Math Methods Med. 2013;2013:358192.

Thysen S, Luyten FP, Lories RJ. Targets, models and challenges in osteoarthritis research. Dis Model Mech. 2015;8(1):17-30.

Torzilli PA, Bhargava M, Chen CT. Mechanical Loading of Articular Cartilage Reduces IL1-Induced Enzyme Expression. Cartilage. 2011;2(4):364-73.

Tuan RS, Chen AF, Klatt BA. Cartilage regeneration. J Am Acad Orthop Surg. 2013;21(5): 303-11.

Ulici V, Alexander PG, Tuan RS. Global gene expression changes following traumatic mechanical impact on bovine articular cartilage. Osteoarthritis and Cartilage. 2014;22:S233-4.

Van Caam A, Davidson EB, Van der Kraan P. TGF $\beta$ Signaling Via ALK1 Requires ALK5 Kinase Activity In Chondrocytes. Osteoarthritis and Cartilage. 2017;25:S167-8.

Van Caam A, Madej W, Thijssen E, Garcia de Vinuesa A, Van den Berg W, Goumans M-J, et al. Expression of TGF $\beta$-family signalling components in ageing cartilage: age-related loss of TGF $\beta$ and BMP receptors. Osteoarthr Cartil. 2016;24:1235-45.

Kraan PM. Differential Role of Transforming Growth Factor-beta in an Osteoarthritic or a Healthy Joint. J Bone Metab. 2018;25:65-72.

van der Kraan PM, Buma P, van Kuppevelt T, van den Berg WB. Interaction of chondrocytes, extracellular matrix and growth factors: Relevance for articular cartilage tissue engineering. Osteoarthr Cartil. 2002;10(8):631-7.

Wang RN, Green J, Wang Z, Deng Y, Qiao M, Peabody M, et al. Bone Morphogenetic Protein (BMP) signaling in development and human diseases. Genes Dis. 2014;1(1):87-105.

Xie J, Fu N, Cai LY, Gong T, Li G, Peng Q, et al. The effects of interleukin- $1 \beta$ in modulating osteoclast-conditioned medium's influence on gelatinases in chondrocytes through mitogen-activated protein kinases. Int J Oral Sci. 2015;7(4):220-31. 